San Jose State University

SJSU ScholarWorks

Master's Theses

Master's Theses and Graduate Research

Fall 2020

\title{
LSTM-enabled Level Curve Tracking in Scalar Fields Using \\ Multiple Mobile Robots
}

Kunj J. Parikh

San Jose State University

Follow this and additional works at: https://scholarworks.sjsu.edu/etd_theses

\section{Recommended Citation}

Parikh, Kunj J., "LSTM-enabled Level Curve Tracking in Scalar Fields Using Multiple Mobile Robots" (2020). Master's Theses. 5157.

DOI: https://doi.org/10.31979/etd.dcjd-92q7

https://scholarworks.sjsu.edu/etd_theses/5157

This Thesis is brought to you for free and open access by the Master's Theses and Graduate Research at SJSU ScholarWorks. It has been accepted for inclusion in Master's Theses by an authorized administrator of SJSU ScholarWorks. For more information, please contact scholarworks@sjsu.edu. 


\title{
LSTM-ENABLED LEVEL CURVE TRACKING IN SCALAR FIELDS USING MULTIPLE MOBILE ROBOTS
}

\author{
A Thesis \\ Presented to \\ The Faculty of the Department of Computer Engineering \\ San José State University
}

\author{
In Partial Fulfillment \\ of the Requirements for the Degree \\ Master of Science
}

by

Kunj J. Parikh

December 2020 
(C) 2020

Kunj J. Parikh

ALL RIGHTS RESERVED 
The Designated Thesis Committee Approves the Thesis Titled

\title{
LSTM-ENABLED LEVEL CURVE TRACKING IN SCALAR FIELDS USING MULTIPLE MOBILE ROBOTS
}

by

Kunj J. Parikh

APPROVED FOR THE DEPARTMENT OF COMPUTER ENGINEERING

\author{
SAN JOSÉ STATE UNIVERSITY
}

December 2020

Wencen Wu, Ph.D.

Department of Computer Engineering

Nima Karimian, Ph.D.

Department of Computer Engineering

Kaikai Liu, Ph.D.

Department of Computer Engineering 


\title{
ABSTRACT \\ LSTM-ENABLED LEVEL CURVE TRACKING IN SCALAR FIELDS USING MULTIPLE MOBILE ROBOTS
}

\author{
by Kunj J. Parikh
}

Autonomous mobile sensor networks are ideal candidates for exploring large-scale unknown fields with tasks ranging from source seeking, level curve tracking, mapping an unknown field, and many more. In this work, we investigate the problem of level curve tracking in unknown scalar fields using a limited number of mobile sensors. The level curve tracking problem has been studied in many applications such as monitoring the propagation of fire boundaries and the algae blooms. We design and implement a long short term memory (LSTM) enabled control strategy for a mobile sensor network to detect and track the desired level curve. We develop on top of existing research which uses cooperative Kalman Filter as part of its motion control strategy. This existing method is theoretically proven to converge. The LSTM enabled strategy has some benefits such as it can be trained offline on a collection of level curves in known fields prior to deployment, where the trained model will enable the mobile sensor network to track level curves in unknown fields for various applications. So we can train using larger resources to get a more accurate model, while we can utilize a limited number of resources when the mobile sensor network is deployed in the production. We design and implement an LSTM-enhanced cooperative Kalman Filter that utilizes the sensor measurements and a sequence of past fields and gradients to estimates the current field value and gradient. We also design an LSTM model to estimate the Hessian of the field. We utilize these estimates of the field characteristics with motion controllers to track the desired level curve in an unknown field with the center of the sensor network. Simulation results show that this LSTM enabled control strategy successfully tracks the level curve using a mobile multi-robot sensor network. 


\section{ACKNOWLEDGMENTS}

I would like to thank my advisor Wencen $\mathrm{Wu}$, for being the greatest advisor in the world. Thank you professor for providing countless advise and for your time for guiding me in the right direction.

I would also like to thank my thesis committee members Nima Karimian and KaiKai Liu for their valuable feedback and advice.

Last, but not least, I would like to thank my family. Without their support, I would have never been able to complete this thesis or pursue my dreams. 


\section{TABLE OF CONTENTS}

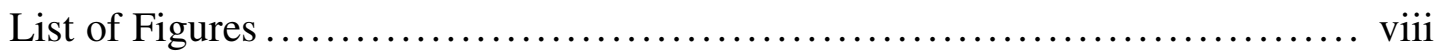

1 Introduction.................................................... 1

2 Literature Review ............................................. 7

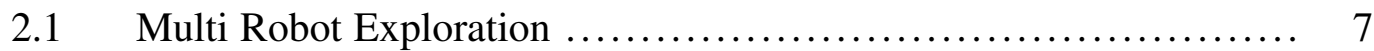

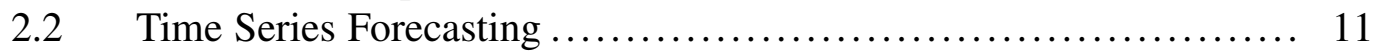

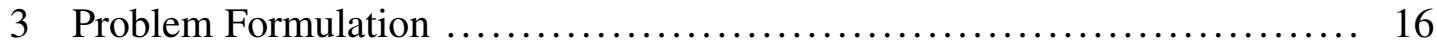

3.1 Scalar Field and Level Curve ................................... 16

3.2 Mobile Sensor Networks ................................ 17

3.3 Three Subproblems .................................... 17

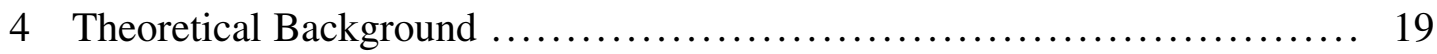

4.1 Multi Robot Exploration ................................... 19

$4.2 \quad$ Cooperative Kalman Filter ................................. 21

4.3 Level Curve Tracking Controller Design ...................... 23

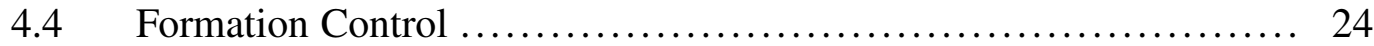

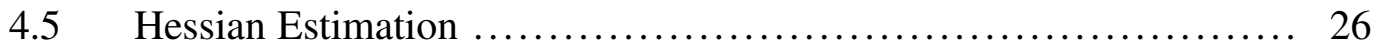

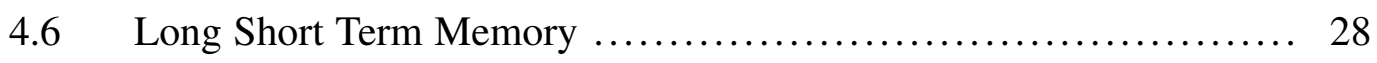

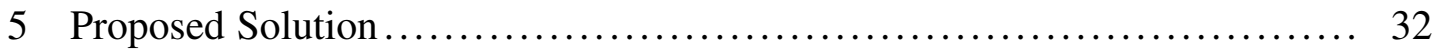

6 Implementation Details ......................................... 40

6.1 Overview ................................................ 40

6.2 Motion Control .............................................. 44

6.2.1 Data Collection.................................... 44

6.2.2 Training Model...................................... 46

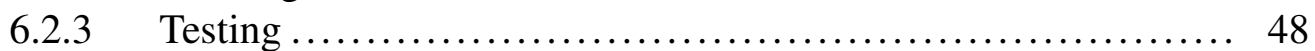

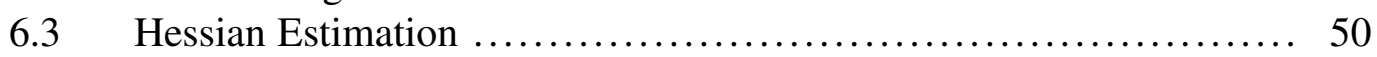

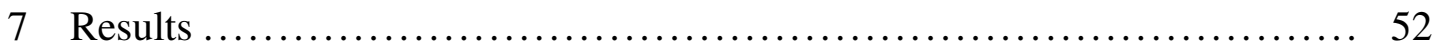

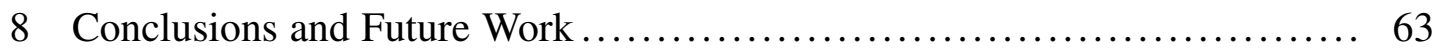

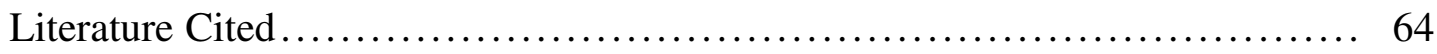




\section{LIST OF FIGURES}

Fig. 1. Motion control components used for level-curve tracking problem.... 19

Fig. 2. Formation center controller design. ....................... 23

Fig. 3. Formation shape arranged around the center................... 25

Fig. 4. A method used to cooperatively estimate the hessian of the field. .... 27

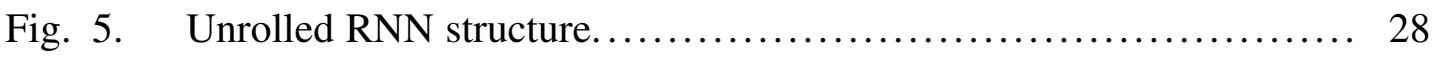

Fig. 6. The LSTM unit cell. .................................. 30

Fig. 7. The proposed motion control flow using estimates generated through LSTM-KF. ........................................ 35

Fig. 8. Single LSTM layer model containing 40 LSTM units. ............. 36

Fig. 9. Independent LSTMs using state and measurements.............. 37

Fig. 10. Combine field and measurements using a fully connected layer. ..... 38

Fig. 11. Field and gradients independent LSTMs.................... 39

Fig. 12. Irregular field tracked by 4 robots........................ 41

Fig. 13. Error metric for evaluating the trained model. ................. 50

Fig. 14. Field traced by robots using traditional Kalman Filter. ............ 54

Fig. 15. Field traced by robots using the predictions by LSTM-KF. ........ 55

Fig. 16. Field and field gradients for actual and predicted trajectory. ........ 56

Fig. 17. $z_{c}$ vs number of iterations for actual and predicted trajectory. ...... 57

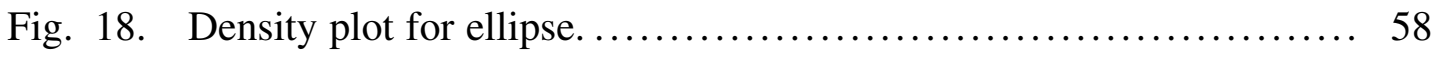

Fig. 19. Correlation with different lags............................ 59

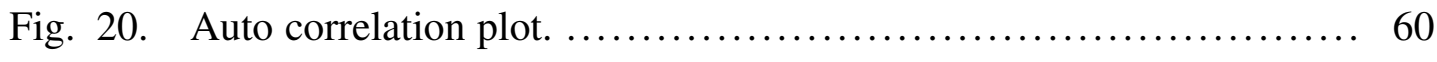

Fig. 21. Trajectory traced by two robot formation around circular level-curve. 61 
Fig. 22. Field and field gradients for actual and predicted 2 robot formation

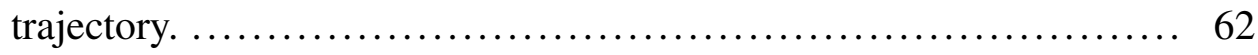




\section{INTRODUCTION}

Individual sensors or a wireless sensors network (WSN) can be deployed in a fixed area to monitor the environment for a long period of time. Example applications of such an arrangement include climate monitoring, monitoring humidity and pressure of redwoods in a forest to analyze the micro-climate around those trees, monitoring vibration of machines in semiconductor fabrication facilities to learn about the machine's health [1]. Taking a step forward we can work with mobile wireless sensor networks (MWSN) like satellites that can monitor charted locations periodically. Other examples of MWSN include attaching sensors to animals to study migratory patterns, sensors attached to manned vehicles, sensors attached to people in hospitals to monitor health signals. Recently, with the development of technology in the field of sensors and unmanned vehicles, a new field of research in sensor networks has emerged. It involves using a swarm of unmanned autonomous robots within the network. Specifically, the group of robots is asked to simultaneously sense an unknown environment, and autonomously decide their trajectory. Some examples of autonomous MWSN include a swarm of unmanned aerial vehicles (UAVs) or unmanned underwater vehicles (UUVs) which are ideal candidates for exploring large-scale environments such as those encountered in the fields of ocean science and meteorology. We can't deploy an unlimited number of robots at static locations to sense the whole large-scale environment (field). As an alternative, we deploy a few robots to study and explore the field.

The autonomous MWSNs are involved in tasks including source seeking, level-curve tracking, mapping an unknown field, and many more. An example of source seeking is climbing gradients of a scalar field [2], [3] to reach the food source, oil spill source, a point at which the temperature is $150 \mathrm{~F}$, etc. An example of level-curve tracking is tracking environmental boundaries [4] as in monitoring the perimeter of a wildfire spreading over 
the land, tracking the perimeter of oil spills, understanding algae blooms, etc. Such a swarm can also sample a large-scale environment in order to chart an unknown field.

MWSNs have to address a few general challenges as described by Cullar et al. [1]. MWSN robots typically have constrained compute hardware resources to perform limited processing on the collected measurements. Depending on the application we need to select sensors to install on the robots; examples of sensors include photocells, fog, temperature, analog-digital converters, micro-electromechanical systems (MEMS), etc. Networking hardware involving radios is a major energy consumer so the design of the network is critical. Another important decision is how will we aggregate data. Do we send raw data or data processed on the edge and at what interval? Next, we have to solve systems challenge, for example using features available in TinyOS operating system. Finally, we need to decide on a power source depending on the consumption and the period of deployment, for example using AA batteries. Autonomous MWSN has additional specific challenges that we address in this thesis. Specifically, we are working in unknown fields so we don't have a way to design a map of the field apriori. We need to design a way to control the trajectory of the autonomous MWSN. Additionally, individual robots of the autonomous MWSN need to cooperate with each other to collect and share the measurements in order to learn the characteristics of the unknown field optimally. Autonomous MWSNs has many benefits. We can finely distribute the sensors as they are not large individually. If designed well and with an appropriate power source or recharge method, they can be deployed for the long term to continuously monitor the environment. Due to their autonomous nature, we don't need anyone explicitly controlling their motion. And in addition, they can be deployed in large scale fields to autonomously explore the unknown field. We use autonomous MWSN for the level-curve or the boundary tracking problem and improve upon existing work. 
There is considerable existing literature which solves some of the challenges involved in the level-curve tracking problem [5], [6], [7]. The robot sensors have the capability to only take measurements at discrete locations and they have limited communication resources to share this information with each other. Zhang and Leonard [6] design a cooperative Kalman Filter which uses the measurements of all the robots to cooperatively estimate the field gradient and the field hessian. They also design control strategies for the swarm trajectory and its shape which uses these estimated field parameters. If we consider the two basic tasks of the level-curve tracking problem, design a control-law which specifies the motion of multiple robots cooperatively, and collect (sense) the data in a cooperative manner, in fact, these two problems are not independent of each other [8]. As an illustration, we need to sense data among multiple robots while cooperating to process the data to calculate the gradient. In parallel, we need to control the motion of each robot cooperatively to maximize data collection. There are additional complexities like time-varying fields that are modeled using advection-diffusion dynamics [9], avoiding obstacles [10], noisy measurements, minimizing update latency, maximizing accuracy by interpolation [11], and many others which we don't discuss in this thesis. We focus on the step which uses cooperatively collected data and state dynamics to estimate the state of the system. Traditionally, a cooperative Kalman Filter is employed to make use of the information dynamics to estimate the state of the system, and it uses instantaneous measurements for this purpose.

In addition to the instantaneous measurements (i.e. data about the current step), we can possibly use a sequence of historical data to infer the next step. Thus we apply machine learning technology to this problem in our current work. Specifically, we apply Long Short Term Memory (LSTM) to study the collected state dynamics to produce state predictions. These state predictions are used with the sensor measurements to generate the state estimates, which are then used to control the trajectory and shape of the swarm 
(formation). In this work, we train the machine learning enhanced system in a set of known fields, and then it is deployed in another set of unknown fields for testing. We keep data collected by the robots for a predefined number of steps and use current and a set of past data to predict the current state estimate. These estimated states are provided to the motion controllers to decide the trajectory of the autonomous MWSN such that it tracks the desired level-curve.

We study the pros and cons of each approach. The traditional cooperative Kalman Filter has benefits. First, we don't need to collect data or train the model separately. Additionally, the convergence of the cooperative Kalman Filter is theoretically proved under certain assumptions. Using LSTM provides us with certain benefits. First, we don't need to explicitly derive the model or the state equations unlike the traditional approach in which we need to model the feedback control which is non-linear and it needs to be designed such that it converges; this needs experience and knowledge of the system. If we follow the machine learning data-driven approach to collect, train, and simulate the system, without knowing a specific model we can accomplish the same task. Second, for complex dynamic fields, these equations are complicated and using input-output data to train an LSTM model is more practical. As a third point, using non-linear machine learning models we can relax the assumptions made in traditional approaches and thereby address more complex fields. Finally, using historical data and machine learning can allow us to reduce the number of required sensors to perform level-curve tracking. Ideally, it can also allow us to use a single robot to track the trajectory instead of a system of robots. There is existing literature we discuss in Subsection 2.2 in which researchers have used LSTM-enhanced Kalman Filter to tackle various challenges such as handling colored noise while taking measurements [12], tracking level-curve without using localization data [13], etc. Given the sensor measurements, we consider both a pure LSTM model and a hybrid model in which the cooperative Kalman Filter is enhanced using LSTM to 
estimate the state. The benefit of the hybrid model is that we can depend on the cooperative Kalman Filter to correct LSTM predictions to make them more accurate, while the benefit of a pure LSTM model is its simplicity.

We make two contributions through this thesis. First, we design and implement an LSTM enabled control strategy for a mobile sensor network to detect and track the desired level-curve. Here we use estimates produced by LSTM-enhanced cooperative Kalman Filter in the controllers used to track the level-curve. This has benefits while working with limited resources. Second, we employ a third LSTM to estimate the hessian for the unknown curve. In the LSTM-enhanced cooperative Kalman Filter, we replace the first two estimating equations in the cooperative Kalman Filter developed in [6] with two LSTM models. This allows us to use multiple steps of historical data to predict (estimate) the next state and error values. We describe this in detail in the subsequent sections. The hessian estimated by the $3^{\text {rd }}$ LSTM is used as an input to the cooperative Kalman Filter and also used in the formation-center model. The benefit of this approach is that we can train the three LSTM models using multiple robots (eg. 4) to more get accurate data. However, when we deploy them in an unknown field we can use fewer robots (eg. 2) to track the curve. We can enable this because we use the pattern information that is available to the LSTM due to its use of long-term historical data. First, we show that if we train the models on a set of field shapes using four robots, and then deploy the models in un-seen field shapes, while still using four robots, we are able to track the curve using four robots. This shows that the model is able to generalize well. Second, we show that instead of using four robots if we use two robots, the model is still able to track the curve, although by trading off some accuracy.

The thesis is organized as follows. In Section 2, we review existing technology and related work. In Section 3, we provide the problem statement. In the next Section 4, we cover some theoretical background, which includes some background about Control 
Theory and Machine Learning LSTM concepts, and the scope of the current project. In Section 5, we first describe our theoretical solution, then we describe the experimental setup. Next, we provide results and analysis in Section 7. Finally, we make some concluding remarks in Section 8. 


\section{LITERATURE REVIEW}

In this chapter, we provide an overview of mobile sensor networks, and we review some multi-robot exploration techniques which are used for level-curve tracking problem. Additionally, we review some time series forecasting methods as applicable to our work of forecasting the state of the multi-robot system.

\subsection{Multi Robot Exploration}

Fixed wireless sensor networks (WSN) are already deployed in many applications [1] like climate temperature, humidity, pressure monitoring in redwood forests, and monitoring motion and vibrations to infer wear and tear in semiconductor fabrication machines. Cullar et al. [1] provides these examples and also gives an overview of the compute (embedded hardware), sensor (MEMS and efficient microsensors), networking (radio energy consumption), system (TinyOS), and power (battery supply) challenges involved in WSNs. They also describe how the data are aggregated across various sensor nodes for analysis. The benefit of WSN, as opposed to wired networks, is they are smaller in size so they can be distributed in a fine manner, and because of lower power requirements, they can be used for long-term monitoring. We are dealing with a special form of WSN - mobile WSN. This is because we are interested in large-scale fields like monitoring temperature in oceans, or monitoring the perimeter of a spreading wildfire.

It is not practical to distribute fixed wireless sensor nodes across large-scale and possibly dynamic fields to form a fixed WSN. Thus, we use a collection of mobile sensor nodes (robots) that forms a mobile wireless sensor network. A mobile wireless sensor network has multiple applications like source seeking, boundary tracking, environmental sampling, etc. Liu et al. [10] describe two algorithms for source seeking applications. First, in which all the robots can communicate with each other, they prove convergence of formation center to the source analytically. Second, in which the robots have limited communication with only neighboring robots, they use a consensus filter to estimate the 
parameters at the formation center. These parameters are then used by controllers to seek the source. Ogren et al. [3] develop a control strategy to perform source seeking. They adapt the sensor array configuration to maximize the gradient climb. A large robot with multiple sensors can't achieve this reconfiguration and so a swarm of robot sensors is superior. In addition to source seeking, another set of applications deals with boundary tracking.

Srinivasan et al. [11] provide a high-level survey of various collaborative boundary tracking techniques. This includes fixed and mobile sensors, networks in which the sensors estimate and track the boundary, and other networks in which sensors cover/surround the boundary, using sensors ranging from in situ, range and remote sensing capabilities, techniques dealing with dynamic boundaries, different field estimation techniques such as calculating contours after sampling an entire field or localized sampling (large-scale fields) close to the boundary and finally different techniques of mobility models ranging from randomized, partially coordinated, approaches that minimize communication cost, swarm-based collective motion and others. Marthaler and Bertozzi [4] apply the 'Snake' algorithm from the field of image segmentation for boundary tracking problems. The algorithm is used to find the boundary of the object in an image. Williams and Sukhatme [7] propose a probabilistic method to map a field using Markov random field to model the system and then they develop control laws to track the level-curve using gradient and hessian estimation. Wang et al. [9] use two cooperating robots to track a dynamic level-curve (plume tracking) in which one robot estimates the gradient, based on measurements, and the second robot patrols the developing plume front. Liu et al. [10] tackle the problem of obstacle avoidance by reframing the cooperative level-curve tracking problem as a constrained optimization problem. They develop an objective function to minimize formation errors. Finally, in addition to the challenges captured by previous level-curve tracking related works, 
Leonard et al. [8] show that the balance between distributed sensing and trajectory planning is a key problem in planning mobile sensor network missions. They define an error metric (measuring statistical uncertainty of the model) to reduce during simulation while sampling data, which captures how well the sensor array samples the field. They also provide control laws for a family of superellipses.

Most of the related works we have discussed talk up to some extent the problem of controlling the trajectory and the formation. Jadbabaie et al. [14] describe a graph theory approach to coordinate the group of robots. In their model, the estimated update of heading for each robot is based on the historical value of its own heading and the headings of nearest-neighbor robots. The set of nearest-neighbor robots dynamically changes as the formation moves along the trajectory. They prove convergence of a model in which the robots are moving with a unit speed but with different headings/directions. Desai et al. [15] describe the control of a nonholonomic mobile robot system using a graph theory approach. They consider one of the robots as a lead robot and model the relationship of other follower robots as a directed graph. They study the transitions between different representations of these control graphs. Orgen et al. [16] address the control of a multi-agent system by first developing Lyapunov functions for individual robots, thereby ensuring formation stability, and then they use the concept of virtual leader to control the trajectory of the formation. Zhang et al. [17] theoretically prove an important result that the motion of the formation center can be decoupled from the motion of formation shape. Modeling the formation as a controlled Lagrangian system and using Jacobi vectors they design shape controllers using Lyapunov functions and independently allow the formation center to climb the gradient or tracks a level-curve. Both the shape and the formation center controllers need accurate estimates of field parameters such as gradient and curvature. Such estimates are produced by filters by observing the sensor measurements. In addition, the controllers need to be designed to optimally collect data for filtering. 
Assuming the unobservable states of the system are related to another process that produces the measurements and which can be observed (Hidden Markov model), there are many filters to accurately estimate the hidden states such as the Zakai equation, Wiener filter, Kalman Filter, and its variants. Olfati-Saber and Shamma [18] describe a way to fuse multiple sensor measurements using consensus filters. Their idea is to run a micro Kalman Filter on the edge sensors, and then a consensus filter is used to combine the results calculated on each node. They also discuss the required size of the sensor network and how it relates to the consensus accuracy for fast signals. Ma et al. [19] approach the consensus problem using a different method. They propose a game-theory approach to the problem, in which they argue that in order to balance a global task with individual interests the problem can be framed as a game. They prove that the game has a Nash equilibrium and they derive the convergence speed to consensus. Rosero and Esteban [5] describe a task combining cooperative source seeking and cooperative level-curve tracking in which they design the formation controllers taking into account limited communication between sensors. In order to estimate the gradients for the controllers, they assume the sensors know each other's relative location and sensor measurements. They model the system of robots using different methods such as single integrator, double integrator, and Linear Time-Invariant system. They also provide stability analysis for each of their algorithms.

Zhang and Leonard [6] develop cooperative Kalman Filter to estimate the state of the system by combining sensor measurements. They also prove the convergence of the cooperative Kalman Filter. In addition, they design controllers to cooperatively determine the formation shape, and another controller to track a level-curve and explore the field. The controllers are designed independently of the Kalman Filter. They only need to meet a set of minimum requirements for the cooperative Kalman Filter to converge. These minimum requirements will have an important role when we discuss two robot systems. 
We study this design in detail in Section 4. Wu and Zhang [20] expand this work to the 3D field by deriving the model for 3D fields and proving its convergence. They apply a modified version of Taubin's algorithm (used in surface smoothing) to estimate the curvature of the surface. They also derive the minimum number of robot sensors required to perform level-curve tracking in a $3 \mathrm{D}$ field.

\subsection{Time Series Forecasting}

Using series or sequence data we can either perform time series analysis or time series forecasting. Time series analysis deals with understanding a data-set by developing mathematical models, and decomposing the time series to understand the underlying processes. This understanding can be used for many purposes, including classifying the time series. Time series forecasting is about extrapolating historical data to predict the future. The primary concern in forecasting is how close predictions are to the actual future values, and not about describing underlying processes. Brownlee [21] provides a very good background on time series, including many traditional statistical methods useful for time series forecasting. Any time series, in general, can be decomposed into four components: level (bias), trend (increasing/decreasing), seasonality (monthly, annual, etc.), and noise. The statistical methods work on these four components. For example, some methods apply transforms on the time series like square-root, log, or a combination, like Box-Cox [22] transforms to model the trend better.

The statistical methods also provide different ways to visualize and better understand the time series. For example, using density plots to understand the distribution of the time series - to see if it is Gaussian distribution. Another example is to plot lag scatter, lag correlation, and autocorrelation plots to understand how much of the time-series history is relevant to predict the current step. We use some of these methods in our analysis to decide the lag-window hyper-parameter. There's another important concept of stationary time series. A time series that has no trend or seasonality is considered stationary, i.e. the 
observations don't depend on time. For a practical dataset like airline passenger dataset trend, the seasonality can be removed to make it stationary. A stationary time series can be modeled using statistical methods like auto regressive moving average (ARMA), which was developed by Box et al. [23]. Here the auto regression (AR) portion models the relationship between the current observation with the historical observations. And, moving average (MA) portion models the relationship between the current observation and the residual error we obtain after applying the moving average model to the historical observations. If the time series not stationary, we can add a integration step which differences a historical observation from the current observation. Thus we can use the auto-regressive integrated moving average (ARIMA) model. This difference step can remove the trend and the seasonality. There are extensions to the ARMA model to handle the multi-variate time series called vector auto regression (VAR) model. For our work, the time-series is multi-variate.

In addition to the statistical methods described above, we can use neural networks to model time series for forecasting. Brownlee [24] discusses various ways in which deep neural networks (DNN) are applied to time series forecasting. The benefits of neural network models are that they are non-linear, so we don't have to worry about stationary data. Additionally, we don't need to make assumptions like Gaussian distribution for the data. They inherently support multiple variables as multiple input features, so we don't need special vector models. The simplest machine learning model is the multi-layer perceptron (MLP), which is an input layer followed by a hidden neural layer, and finally an output layer. Here, the historical time-series data is simply passed as extra features to the input layer. For example, if we have 3 features at each time-step, and we are considering a lag-window of 4, for MLP we provide 12 features as input to predict 3 features. We consider a similar model containing fully connected dense layers as part of our experiments. Similar to the MLP, we can use convolutional neural network (CNN), in 
which we provide historical data as extra features, and the trained CNN's weights assign importance to features. Recurrent neural networks (RNN), specifically LSTM were designed specifically for sequence data, such as time series, a series of words in a sentence for language translation, a series of images as in the video, etc. An LSTM contains a series of unit cells which are described in detail in Subsection 4.6. These unit cells maintain a state that is passed on to the next unit cell, in which some information is added, removed or updated, and the modified state is passed on to the subsequent unit cells. This state models the series, and remembers the effect of historical data on current prediction. Traditional RNNs suffered from a problem, in which they can't learn long term dependencies. LSTMs are designed to solve this problem, and they have shown considerable success in many problems like language translation [25], speech recognition [26], video recognition and narration [27], time series forecasting [28] [29] and many others. LSTMs can also be arranged to create more complex networks like stacked LSTM, in which two LSTM layers (each containing multiple units) are connected back to back. This enables it to learn both the short-range and the long-range patterns. Examples of even more complex LSTM networks include bidirectional LSTM (useful for translation to see both the preceding and the following words), CNN-LSTM, and ConvLSTM (which are useful for video analysis to extract image features using CNN and then use LSTM on those features). We consider both a simple LSTM layer and a stacked LSTM for this thesis.

There's a lot of existing research in the field of motion control and state estimation using neural networks. Yang and Luo [30] propose a neural network model for complete coverage path planning problems including obstacle avoidance. Here, they use inputs from the dynamic landscape and the previous location of the robot to decide the robot's trajectory. Choi et al. [12] tackle the problem of simultaneous localization and tracking (SLAM) using the neural network aided extended Kalman Filter (NNEKF). For colored 
noise, the extended Kalman Filter diverges, so they model the noise using a neural network, and use a combination of EKF and NN to control the motion. In their proposal, the NN is trained online, so it can handle un-modeled dynamics too. Krishnan et al. [31] propose deep Kalman Filters to deal with the problems faced while modeling non-linearity using standard Kalman Filter, and to deal with long-range temporal interactions. They assume the distribution of the latent state is normal and can be modeled using a sequence of actions and observations. They parameterize these functions and train the parameters using deep neural networks to effectively learn a broad range of Kalman Filters. Doshi [32] applies a similar concept to estimate the pose of the vehicle using video data. Here, they use $\mathrm{CNN}$ to extract the features, and RNN to estimate the velocity. They show better performance than the multi-state constraint Kalman Filter. Masti and Bemporad [33] use another machine learning model - deep autoencoders - as a way to learn nonlinear state-space representation given the input/output dataset. Zhang et al. [34] apply deep RNNs for state estimation (non-linear dependency) and forecasting in the field of power system. This has traditionally been solved using extended Kalman Filters or vector auto regression (VAR). Coskun et al. [35] present the LSTM Kalman Filter (LSTM-KF) design for pose identification, for which traditionally a Kalman Filters have been used for regularization. But for pure a Kalman Filter the models need to be designed apriori. Their LSTM-KF design replaces three matrices with LSTMs: the state transition matrix, the process covariance matrix, and the measurement covariance matrix. Then they use the output of the three LSTMs in KF's gain and update equations, in which they also use observed measurements. Zhang et al. [36], [13] also employ a similar LSTM-KF design for problems related to collecting data in a 2D spatio-temporal varying field for underwater gliders. They track a level-curve without using the localization data. For this work, we use a similar LSTM-KF design to the problem of estimating state to perform level-curve tracking. We use the historical data and the observed field values to estimate 
the field gradients. These are used in the motion controllers to track the desired level-curve. We describe this design in detail in Section 5. 


\section{PROBLEM FORMULATION}

In the following subsections, we describe the components tha. Theye up tproblem statement. Fir using thest, we define what we mean by a scalar field and a level-curve we desire to track. Second, we describe the mobile sensor network (or the swarm of robot sensors), we define some related terms like formation center and format. Theseape, and we discuss the assumptions we make relating to the robot's capabilities. Finally, using the definition of the level-curve and mobile sensor network, we define the three sub-problems we work on in this thesis: estimating field and gradient, designing a controller for the formation center, and designing an independent controller for the shape of the formation.

\subsection{Scalar Field and Level Curve}

We can model temperature in the ocean or in a wildfire using a scalar function of three dimensions. In order to simplify the problem, we assume this function is a static field as opposed to a time-varying dynamic field. Then, temperature at each point in the

ocean can be represented as a function over $R^{3}$, i.e. $f(x, y, z)$. In this work, we focus on 2-dimensional fields so we consider functions of the form $f(x, y)$. This function (or temperature field) is not known apriori, but our problem is also not to fully estimate this field $\forall(x, y) \in R^{2}$. We assume the field is smooth and that the field can be approximated using second-order variations. Thus the field characteristics at any location $(x, y)$ can be defined using the field value, the field gradient, and the curvature of the field (i.e. hessian) at that location. We denote the three characteristics of field by $z(r), \nabla z(r)$ and $H(r)$ where $r$ is a $(x, y)$ point in 2D space (i.e. $\left.r \in R^{2}\right)$. We assume that the field is smooth, its gradient is well-defined and bounded by a min and max value at all locations, i.e.

$\nabla_{z_{\text {min }}}<\nabla_{z}(r)<\nabla_{\text {max }}, \forall r \in R^{2}$

Let us consider the definition of a level-curve. A level set $L_{c}(f)$ of a function $f$ with $n$ variables is defined as a set of inputs for which the function takes a constant value $c$, i.e. $L_{c}(f)=\left\{\left(x_{1}, . ., x_{n}\right) \mid f\left(x_{1}, . ., x_{n}\right)=c\right\}$. For a two dimensional field (i.e. $n=2$ ) this level 
set is called a contour or a level-curve. Our problem is to find and track a level-curve given a desired constant value of the field. To again note, the field is not known to us, the only capability we have is to measure the field value at a given location. That is, we can measure $z_{1}$ at $\left(x_{1}, y_{1}\right)$ such that $z_{1}=f\left(x_{1}, y_{1}\right)+\varepsilon$. The measurements are not accurate so we add the $\varepsilon$ term to model the error. In addition, we deal with large-scale fields such as oceans, large wildfires, etc. so we can't sample all possible locations to measure the field.

\subsection{Mobile Sensor Networks}

With these constraints, we consider a scenario in which we deploy $N$ robots in this field, and each robot has a sensor to measure the field at its own location. We also call this collection of robots a swarm or a formation. We make the following three assumptions regarding the robot sensor's capabilities. First, we assume that the measurements are taken at discrete intervals, and we denote these local measurements by individual robots as $z_{i}$ where $i \in\{1, . ., N\}$. Second, we assume the robots can communicate with each other, so they can share their local field measurements with each other. Third, in this current work, we also assume that the robots know the relative locations of each other, which they can also communicate with others. This group of mobile robots which can measure the field and communicate with each other forms what we call the mobile wireless sensor

network. We denote individual robot locations as $r_{i}$ where $i \in\{1, . ., N\}$ and $r_{i} \in R^{2}$, and we denote the center of the formation using $r_{c}$, where $r_{c}=\sum\left(r_{i}\right) / N$. Any or each of the robots can then use the collection of measured local field values and corresponding relative locations to estimate the characteristics of the overall field using methods we will describe in subsequent sections.

\subsection{Three Subproblems}

The first problem is to accurately estimate $z(r), \nabla z(r)$, and $H(r)$ using the collection of measurements $z_{i}$ and corresponding locations $r_{i}$ close to $r$. We take the center $r_{c}$ as the point close to all the individual $r_{i}$ to represent the formation. The second problem is to 
design a motion controller, which uses these estimates at the center $z_{c}, \nabla z_{c}$ and $H_{c}$, so that the formation center can detect and track a level-curve with the desired field value, i.e. design a controller for the formation center so that $z_{c}=z_{\text {desired }}$. The third problem is how to control the motion of individual robots. Zhang et al. [17] have shown using the Jacobi transforms we can decouple the motion of the formation center $r_{c}$ from the motion of the formation shape (i.e. individual $r_{i}$ in relation to each other). They use this to design a controller to control the formation shape, which is independent of the controller designed to ensure that the formation center tracks the level-curve. The individual locations decided by this controller will affect the measurements taken and thus the field estimate at the next step, so this controller needs to be designed to get optimal field estimates.

Therefore, we end up with three main components to achieve motion control to track a level-curve: a way to estimate field characteristics, a controller to control the formation center, and a controller to control the formation shape. In Section 4 we describe these three components in detail, and in Section 5 we propose a method that utilizes historical measurement data instead of just the current measurements and we discuss its benefits. 


\section{THEORETICAL BACKGROUND}

We first describe an overview of the method used to model the robot measurements to estimate the field and the field gradients, which make up the state of the system. Next, we describe the cooperative Kalman Filter used to estimate the state. After this, we describe a method to cooperatively estimate the hessian of the field. Then we describe the two controllers used to plan the motion. Fig. 1 describes how the components fit together to use robot measurements to track the level-curve. Finally, we describe a neural network used for current work.

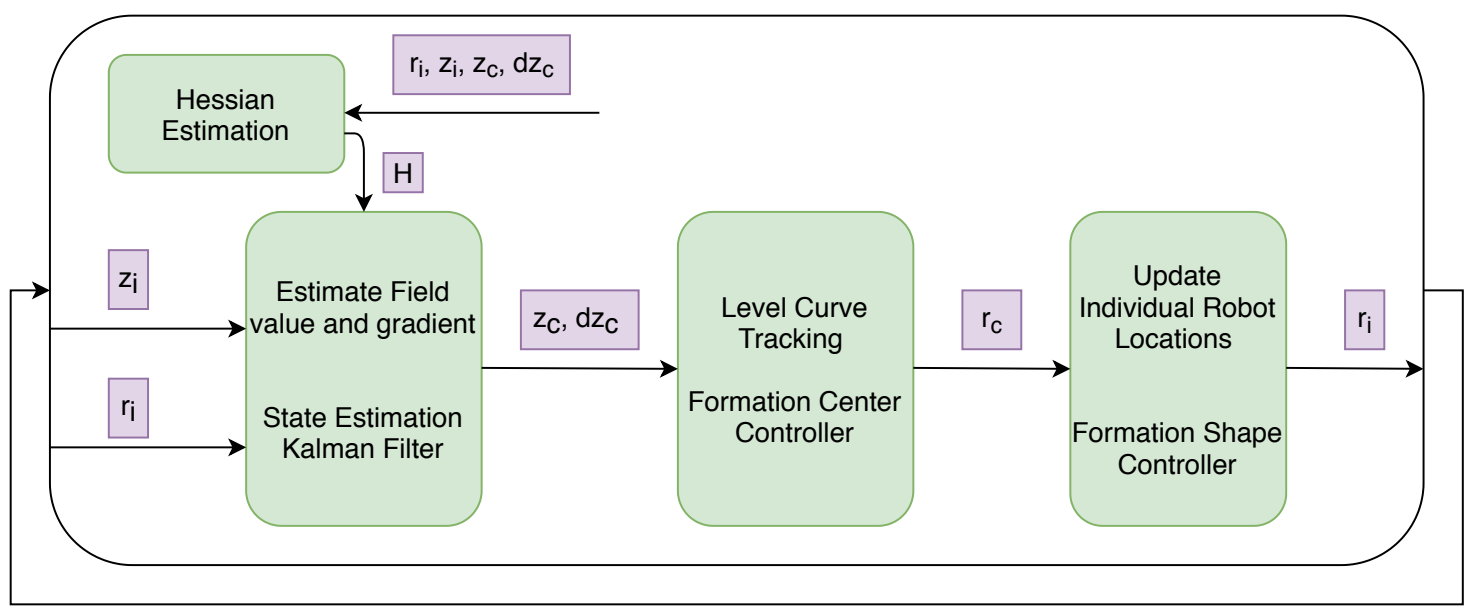

Fig. 1: Motion control components used for level-curve tracking problem. Cooperative Kalman Filter and hessian estimation provides estimates of field characteristics, which are used by the formation center controller and formation shape controller to update the robot locations to track the level-curve.

\subsection{Multi Robot Exploration}

We need a way to estimate field characteristics $z_{c}, \nabla z_{c}$ and $H_{c}$, given robot measurements $z_{i}$ and $r_{i}$. The unknown field can be modeled as a Hidden Markov Model (HMM) system of which we observe measurements in the form of field measurements. This system has a latent state which transitions with time, and the hidden process produces measurements corresponding to this latent state which we observe. The latent 
state is composed of field value $z(r)$ and the field's gradient in $\mathrm{X}$ and $\mathrm{Y}$ directions $\nabla_{x} z(r)$ and $\nabla_{y} z(r)$. We observe the measurements and use them to estimate the subsequent state of the system, i.e. to estimate the field values and the field gradients.

Zhang and Leonard [6] describe the dynamics of this modeled system. They first consider the field observations having measurement errors. Then they approximate the actual field value at the individual robot locations as a function of the formation center. They combine these to relate observed measurements to the system state consisting of $z_{c}$ and $\nabla z_{c}$. Lastly, they describe the state transition as the formation tracks the level-curve. An overview of the equations is presented below. We model the measurement $p$ taken by each robot $\mathrm{i}$ at time $\mathrm{k}$ as:

$$
p_{i, k}=z_{i, k}+w_{i, k}+n_{i, k}
$$

where $z$ is the actual value of the field, $n$ is random noise, $w$ is spatially correlated noise.

Assuming the field is smooth with respect to location $r$, we can approximate $z$ around formation center $r_{c, k}$ by its second-degree Taylor expansion.

$$
z_{i, k} \approx z_{c, k}+\left(r_{i, k}-r_{c, k}\right)^{T} \nabla z_{c, k}+\frac{1}{2}\left(r_{i, k}-r_{c, k}\right)^{T} \nabla^{2} z_{c, k}\left(z_{i, k}-r_{c, k}\right)
$$

where $z_{c, k}$ is the field value at the formation center $r_{c, k}, \nabla z_{c, k}$ is the gradient of the field at $r_{c, k}, \nabla^{2} z_{c, k}$ is the second derivative of the field at $r_{c, k}$, and $z_{i, k}$ is the field value at the $i^{t h}$ robot's position.

The state of the system can be defined as $\mathbf{s}_{k}=\left[z_{c, k}, \nabla_{x} z_{c, k}, \nabla_{y} z_{c, k}\right]$. The measurements $p_{i, k}$ of all the robots can be combined as a vector $p_{k}$. Similarly, combining noise elements for each robot's measurement, we get vectors $w_{k}$ and $n_{k}$. The second derivative of the field $\nabla z_{c, k}$ can be estimated using hessian $\mathbf{H}_{c, k}$. The error in estimating the hessian is represented by $e_{k}$. 
Using these notations and combining (1) and (2), we get,

$$
p_{k}=C_{k} s_{k}+D_{k} \mathbf{H}_{c, k}+D_{k} e_{k}+w_{k}+n_{k}
$$

where

$$
C_{k}=\left[\begin{array}{cc}
1 & \left(r_{1, k}-r_{c, k}\right)^{T} \\
\cdot & \cdot \\
\cdot & \cdot \\
1 & \left(r_{N, k}-r_{c, k}\right)^{T}
\end{array}\right],
$$

and $D_{k}$ is a $N x 4$ matrix whose $i^{t h}$ row is defined by $\frac{1}{2}\left(\left(r_{i, k}-r_{c, k}\right) \otimes\left(r_{i, k}-r_{c, k}\right)\right)^{T}$.

As the formation moves, the state $\mathbf{s}$ evolves following the below equation,

$$
s_{k}=A_{k-1}^{s} s_{k-1}+h_{k-1}+\varepsilon_{k-1}
$$

where

$$
\begin{gathered}
A_{k-1}^{s}=\left[\begin{array}{cc}
1 & \left(r_{c, k}-r_{c, k-1}\right)^{T} \\
0 & I_{2 x 2}
\end{array}\right], \\
\left.h_{k-1}=\left[\begin{array}{ll}
0 & E\left[H_{c, k-1}\left(r_{c, k}-r_{c, k-1}\right)\right.
\end{array}\right]^{T}\right]^{T},
\end{gathered}
$$

and $\varepsilon_{k-1}$ is noise (independent of $n$ or $w$ ). This noise $\varepsilon_{k-1}$ accounts for the positioning errors, hessian estimation error and error caused by omitting higher order terms in the Taylor expansion.

\subsection{Cooperative Kalman Filter}

Knowing the model for measurements and the state transition model, we can combine these to estimate the subsequent state using the measurements. Using Equations (3) and (4) we can design a cooperative Kalman Filter. The convergence of this Kalman Filter is proved by Zhang and Leonard [6]. The Kalman Filter contains five equations: The first equation provides one-step state prediction using previous state $s_{k-1}$ and hessian. The 
definitions of $A_{k-1}^{s}$ and $h_{k-1}$ are the same as in previous Subsection 4.1.

$$
s_{k(-)}=A_{k-1}^{s} s_{k-1(+)}+h_{k-1}
$$

The second equation estimates the error covariance in the one-step prediction. Here $P_{k-1}$ is the updated error covariance in the last iteration,

$$
P_{k(-)}=A_{k-1}^{s} P_{k-1(+)} A_{k-1}^{s T}+M_{k-1}
$$

The third equation calculates the optimal gain using the error covariance estimated above. The matrices $C_{k}, D_{k}$ are defined in the Subsection 4.1. $U_{k}=E\left[e_{k} e_{k}^{T}\right]$ and $R_{k}=E\left[n_{k} n_{n}^{T}\right]$, where $e_{k}$ and $n_{k}$ are defined in Equation (3)

$$
K_{k}=P_{k(-)} C_{k}^{T}\left[C_{k} P_{k(-)} C_{k}^{T}+D_{k} U_{k} D_{k}^{T}+R_{k}\right]^{-1},
$$

The fourth and the fifth equations use the robot measurements $p_{k}$ and the calculated optimal gain $K_{k}$ to obtain updated the estimates of state and error covariance $s_{k(+)}$ and $P_{k(+)}^{-1}$.

$$
\begin{gathered}
s_{k(+)}=s_{k(-)}+K_{k}\left(p_{k}-C_{k} s_{k(-)}-D_{k} H_{c, k}\right), \\
P_{k(+)}^{-1}=P_{k(-)}^{-1}+C_{k}^{T}\left[D_{k} U_{k} D_{k}^{T}+R_{k}\right]^{-1} C_{k} .
\end{gathered}
$$

In summary, we first predict the state using the equation for $s_{(-)}$, then we calculate the predicted measurement $P_{(-)}$. We use actual measured value and calculate the Gain $K$. Then we update our predicted estimates to $s_{(+)}$and $P_{(+)}$. And thus we obtain estimates of field value $z_{c}$ and field gradient $\nabla z_{c}$ at $r_{c}$. 


\subsection{Level Curve Tracking Controller Design}

We can use the field characteristics at the formation center to find a new formation center, such that the field value at the new formation center is the same as the current field value at the formation center. That is, given estimates of $z_{c, k}, \nabla z_{c, k}$ and $H_{c, k}$, we can find $r_{c, k+1}$ such that $z_{c, k}=z_{c, k+1}$, where $k$ is the current step and $k+1$ is the next step. Using the new formation center calculated thus ensures that we track the same field values (i.e. the level-curve) using the formation center. We use the controller described in Zhang et al. [8] which uses estimated $z_{c}, \nabla z_{c}, H_{c}, z_{\text {desired }}$ and the current trajectory of the center $r_{c}$ to update the trajectory. They model the formation as a unit mass Newtonian particle and represent the system in Frenet-Serret form which allows them to separate the steering control from the speed control. As described in the Fig. 2, at any point along a level-curve, we can define an angle $\phi$ formed between the direction tangent to the field $x_{1}$ and the current heading of the formation center $x$. Here $y_{1}$ is the same as the direction of the field gradient $\nabla z_{c}$ estimated earlier, and $y$ is the direction orthogonal to current heading $x$.

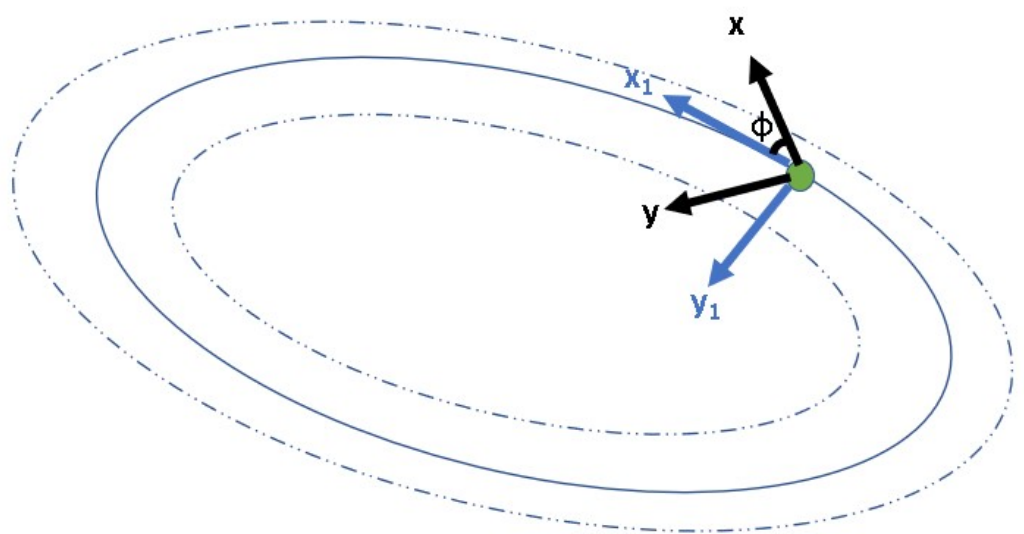

Fig. 2: Formation center controller design. Angle $\phi$ between the heading of the formation (represented by $\vec{x}$ ) and the tangent to the level-curve (represented by $\overrightarrow{x_{1}}$ ) is used by the control law as shown in Equation (10). Direction of field gradient $\overrightarrow{y_{1}}$ is used to determine tangent $\overrightarrow{x_{1}}$. 
Using the angle $\phi$ described above and assuming unit speed, the steering control law can be described by the following equation,

$$
u_{c}=\kappa_{1} \cos \phi+\kappa_{2} \sin \phi-2 \tilde{f}(z)|| \nabla z \| \cos ^{2}\left(\frac{\phi}{2}\right)+K_{4} \sin \left(\frac{\phi}{2}\right) .
$$

Here $\kappa_{1}=-\frac{x_{1}^{T} \nabla^{2} z x_{1}}{\|\nabla z\|}, \kappa_{2}=\frac{x_{1}^{T} \nabla^{2} z y_{1}}{\|\nabla z\|}$ in which $\|\nabla z\|$ is obtained using estimated field gradient $\nabla z_{c} . \nabla^{2} z_{c}$ is obtained using hessian estimation. $\tilde{f}(z)=0$ if the formation center $r_{c}$ is on the level-curve, otherwise it models the external force which drives the formation towards the desired field value $z_{\text {desired }} . K_{4}$ is a constant gain parameter we can tune. The calculated steering control can be used to update the heading of the formation center, and calculate the new formation center $r_{c, k+1}$.

\subsection{Formation Control}

We use a controller described by Zhang and Leonard [6] to control the shape of the formation. This controller is designed independently of the formation center controller which tracks the level-curve. They derive the guidelines for designing formation shape controller which yield successful cooperative Kalman Filters. For $N \geq 3$, we can fix the formation shape, but we need to ensure that the formation is not singular. That is, don't arrange all the robots in a straight line or at a point. In this work, for a network of four robots $(N=4)$ we specify the desired relative distance between the robots. We attain this relative distance when we achieve a steady-state, in which the four robots form a static formation taking four corners of a rhombus. Fig. 3 describes the formation for $N=4$ robots in the steady-state. Here, $2 b$ is the desired steady-state distance between $r_{1}-r_{2}$, and $2 a$ is the distance between $r_{3}-r_{4} \cdot r_{2}-r_{1}$ are oriented along fixed lab frame direction $e_{2}$, and $r_{3}-r_{4}$ is oriented along $e_{1}$. Additionally, $e_{2}$ is orthogonal to $e_{1}$. The steady-state values $a$ and $b$ can be numerically calculated by solving the Riccati equation, which minimizes steady error covariance $P_{\infty}$ of the cooperative Kalman Filter. Given the 
symmetry of the formation shape and the values $a_{k}$ and $b_{k}$ (where $a_{k} \rightarrow a, b_{k} \rightarrow b$ in the steady-state), the matrices $C_{k}$ and $D_{k}$ can be reduced to a simpler form.

$$
\begin{gathered}
C_{k}=\left[\begin{array}{ccc}
1 & -b_{k} & 0 \\
1 & b_{k} & 0 \\
1 & 0 & a_{k} \\
1 & 0 & -a_{k}
\end{array}\right], \\
D_{k}=\left[\begin{array}{cccc}
b_{k}^{2} & 0 & 0 & 0 \\
b_{k}^{2} & 0 & 0 & 0 \\
0 & 0 & 0 & a_{k}^{2} \\
0 & 0 & 0 & a_{k}^{2}
\end{array}\right] .
\end{gathered}
$$

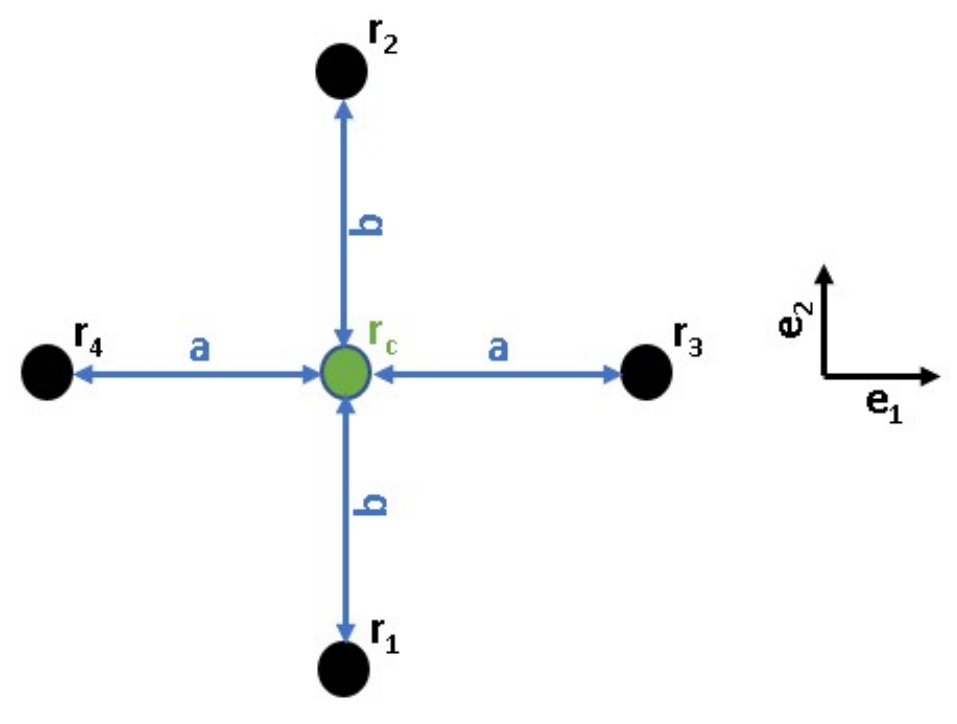

Fig. 3: Formation shape arranged around the center. Four robots $r_{1}, r_{2}, r_{3}$ and $r_{4}$ are arranged around the center $r_{c}$, such that $\left(r_{1}-r_{2}\right) \perp\left(r_{3}-r_{4}\right)$. The relative distance between $r_{3}, r_{4}$ and $r_{c}$ is denoted by $a$, between $r_{1}, r_{2}$ and $r_{c}$ as $b$. The values $a$ and $b$ are derived to optimally collect data for cooperative Kalman Filter and ensure its convergence.

For a network of two robots $(N=2)$, we can similarly derive a steady state value of distance between two robots $a_{k}$, but the controller is slightly different. In order to keep all the matrices non-singular we need to rotate the two robots around the center. Intuitively, 
we need to measure enough information to be able to calculate gradients in two directions, so we need to rotate the two robots around the formation. Using less number of robots also affects the accuracy of how close the formation is able to track the trajectory.

Using this formation shape controller, once we know the new formation center $r_{c, k+1}$ using the formation center controller, we can calculate new locations of individual robots $r_{i, k+1}$. We need special care in calculating these new individual $r_{i, k+1}$ to ensure that the new measurements the robots take $z_{i, k+1}$ will provide a good estimate of the field characteristics at the new formation center $z_{c, k+1}, \nabla z_{c, k+1}$. We ensure this by reducing the Kalman Filter estimate's error covariance while deriving steady state values of $a$ and $b$. Repeating this procedure iteratively, we will end up with a sequence of formation centers $r_{c}$. This sequence should be one of the desired level-curves represented by a set of points $\left\{(x, y): f(x, y)=z_{\text {desired }},(x, y) \in \mathbf{R}^{2}\right\}$, where $z_{\text {desired }}$ is a user-defined constant.

\subsection{Hessian Estimation}

The cooperative Kalman Filter and the formation center controller requires the second derivative $\nabla^{2} z_{c}$ ), which can be obtained by estimating the hessian $H_{c}$ of the field using sensor measurements. Fig. 4 describes the algorithm used by Zhang and Leonard [6] to estimate hessian of the field using a formation of four robots. In the Fig. $4, r_{1}, r_{2}, r_{3}, r_{4}$ are the sensor robots moving in a formation around the center $r_{c}$. First, find centers of robots taken three at a time, i.e. find point $r_{E}$ which is the center of $r_{1}, r_{2}$ and $r_{4}$. Similarly, find $r_{F}$ which is a center of $r_{1}, r_{2}$ and $r_{3}$. 


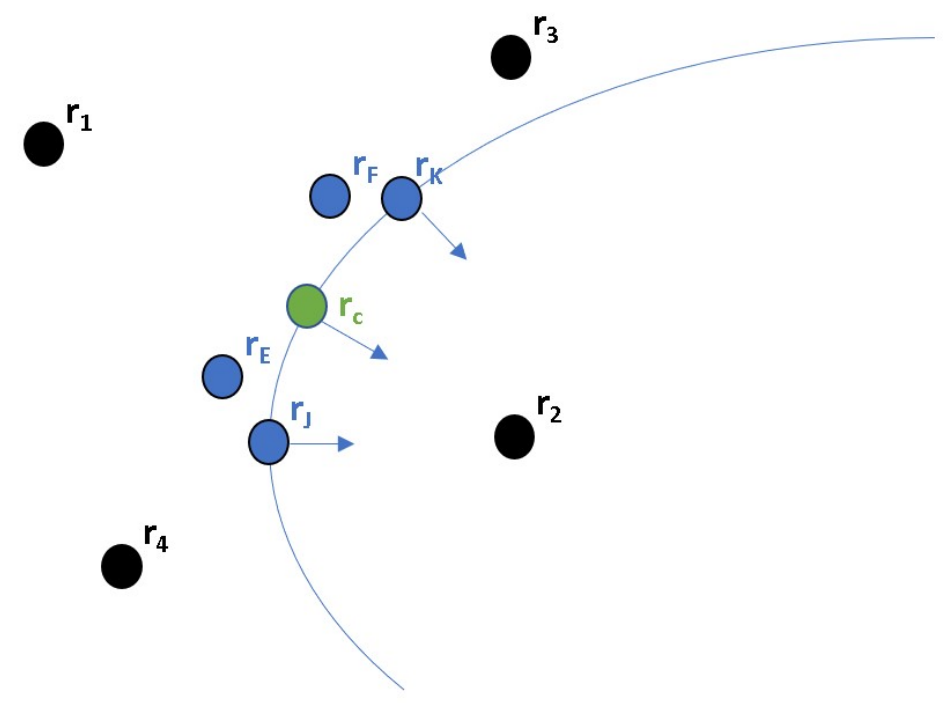

Fig. 4: A method used to cooperatively estimate the hessian of the field. Four robots $r_{1}, r_{2}, r_{3}$ and $r_{4}$ are arranged around the center $r_{c}$ similar to Fig. 3. $r_{E}$ is the center of sensors $r_{1}, r_{2}$ and $r_{4} . r_{F}$ is the center of sensors $r 1, r 2$ and $r_{3} . r_{J}$ and $r_{K}$ are on the desired level-curve along with $r_{c} . r_{J}$ and $r_{K}$ are determined using field and gradients at $r_{E}$ and $r_{F}$ respectively.

Using the field measurements at the robot locations $z_{i}$ and the previous hessian estimate, we can calculate the field and the field gradient at $r_{E}$ and $r_{F}$.

$$
z_{i}=z_{E}+\nabla z_{E} *\left(r_{i}-r_{E}\right)+\frac{1}{2}\left(r_{i}-r_{E}\right)^{T} H^{P}\left(r_{i}-r_{E}\right), \forall i=1,3,4 .
$$

We can solve for three variables $z_{E}, \nabla_{X} z_{E}$ and $\nabla_{Y} z_{E}$ in the above set of equations. Similarly, we can obtain $z_{F}$ and $\nabla z_{F}$. Using $z_{E}$ and $\nabla z_{E}$, we can find a point $r_{J}$ on the level-curve and again calculate the gradient $\nabla z_{J}$ using the previous hessian $H_{c}^{P}$. Similarly find $r_{K}$ and $\nabla z_{K}$. Now that we have gradients at three points along the level-curve $\nabla z_{K}$, $\nabla z_{c}$ and $\nabla z_{J}$, we can estimate the hessian at $r_{c}$. We can use this estimated hessian $H_{c}$ and repeat the steps using this hessian, instead of previous hessian estimate $H_{c}^{P}$ to get a even 
better estimate of hessian. Thus, the algorithm described above becomes a iterative numerical algorithm.

\subsection{Long Short Term Memory}

RNN are a type of neural network in which the directed graphs are used to model a temporal sequence of data. RNN maintains an internal state to learn the sequence input to the network. Fig. 5 [37] shows the structure of a RNN. he left side shows $x$ given as input to the RNN unit cell, $h$ is the hidden layer, and $o$ is the output. Matrices $U, W$ and $V$ contain the weights learned when we train the RNN. They control how the data is transformed while passing from the input layer to the hidden state $U$,. That is, how the internal state is used for the next time step $V$, and how the internal state is transformed to the observed outputs $W$. The RNN can be unfolded along the time axis to understand how the input sequence $x_{t-1}, x_{t}, x_{t+1}$ is transformed in the hidden layer. It also explains how the hidden layer connects across the different time-steps $h_{t}$. Finally, it also explains how the sequence inputs and hidden layer states are related to observed output sequence $o_{t}$.
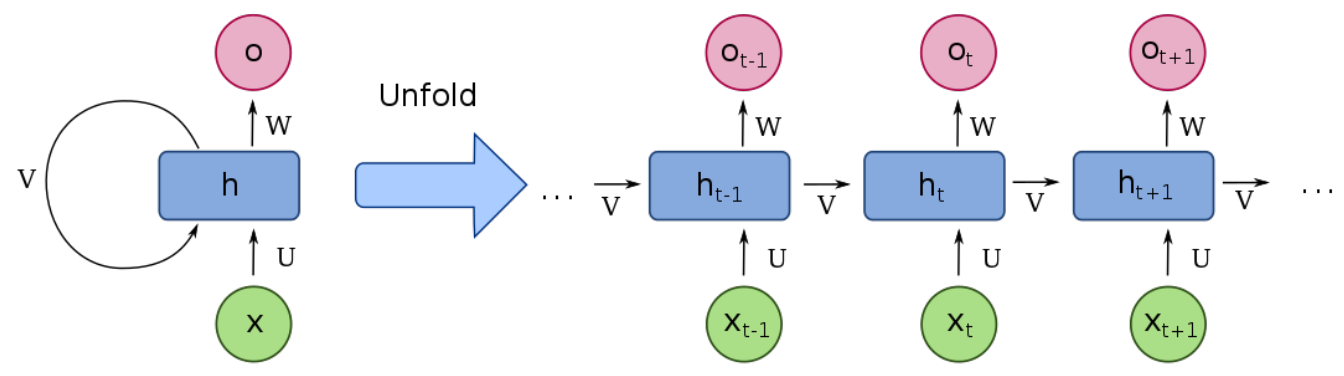

Fig. 5: Unrolled RNN structure. The unrolled (or unfolded) RNN shows how the information flows for different time-steps. The weights of the RNN unit cell doesn't change for different time steps. At time-steps $t-1, t$ and $t+1, x_{t-1}, x_{t}$ and $x_{t+1}$ shows the external input, $o_{t-1}, o_{t}$ and $o_{t+1}$ denotes the external output, and $h_{t-1}, h_{t}$ and $h_{t+1}$ represents the internal state respectively. Matrices $U$ and $W$ represent the input and output weights respectively. Matrix $V$ contains the weights that control how state information from one time-step is passed onto the next time-step. 
RNNs don't handle long-term dependencies very well. That is, the older the data gets, the less affect it has on the current output. Hochreiter and Schmidhuber [38] introduced the design for LSTM in 1997. LSTM is a type of RNN that focuses on retaining long-term past information/learning by maintaining state, and using the saved state with current input at any step. LSTMs do this by introducing various gates in the LSTM unit cell. Intuitively, the gates help to short through the unimportant time steps, and thus remember the data longer.

Fig. 6 [39] shows the structure of one LSTM cell and how it is connected in a chain. The LSTM unit cell maintains the cell state $C_{t}$ in addition to what the RNN unit cell does. The unit cell takes the inputs $X_{t}$, the previous cell state $C_{t-1}$ and the previous output $h_{t-1}$. After performing some operations (described next) it generates the current output $h_{t}$ and the updated cell state $C_{t}$. In the figure, we can see the LSTM is composed of 4 gates in orange. Olah [40] provides a good overview of the LSTM unit cell structure. The first gate $f$ is the forget gate. It decides how much information from the previous state $C_{t-1}$ needs to be passed on to the subsequent states $C_{t}$. Second is the input gate $i$. It decides which of the state values $C_{t-1}$ needs to be updated by the current stage's input $X_{t}$. The third gate $C^{\prime}$ scales the current input $X_{t}$ before passing it in to update the state $C_{t}$. The last gate is the output gate $o$. It takes the current input $X_{t}$ and the updated state $C_{t}$ and produces the current stage's output $h_{t}$. The updated state $C_{t}$ and the output $h_{t}$ are given as input to the next stage. Here, $\sigma$ is the sigmoid function $\sigma(x)=\frac{e^{x}}{e^{x}+1}$ having the range $[0,+1] . \tanh$ is the hyperbolic tangent function $\tanh (x)=\frac{e^{2 x}-1}{e^{2 x}+1}$ with the range $[-1,+1]$, 


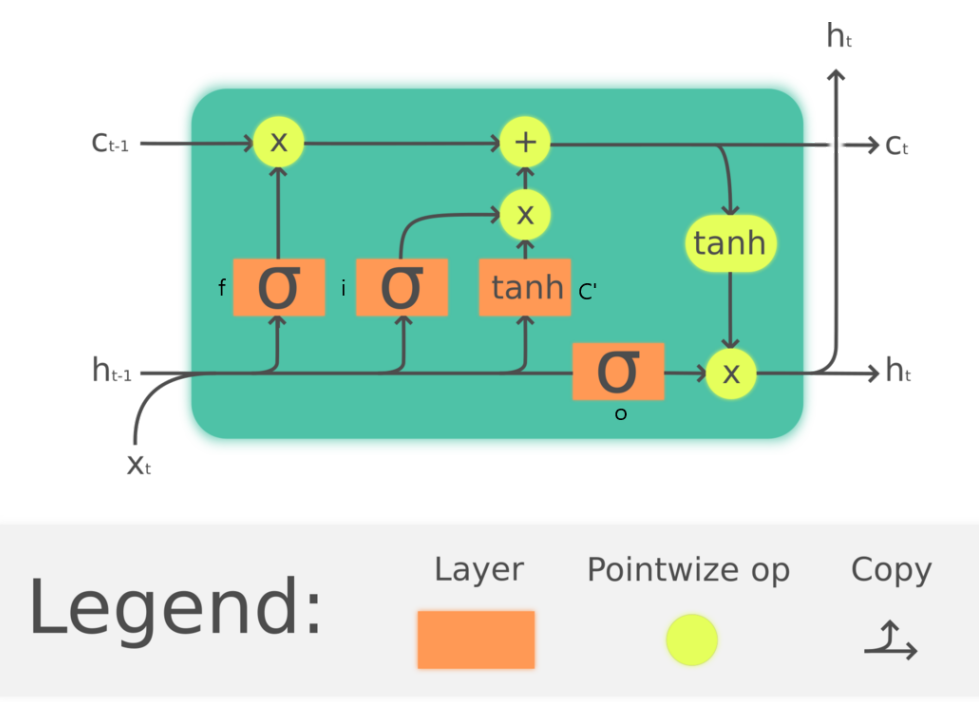

Fig. 6: The LSTM unit cell. $x_{t}$ and $h_{t}$ represents external input and output at the time-step $t$. $h_{t-1}$ denotes the output of previous time-step which is passed in as input for time-step $t$. $C_{t-1}$ and $C_{t}$ represents the cell state at $t-1$ and $t$ respectively. Forget gate $f$ controls, depending on the inputs $x_{t}, h_{t-1}$, how much of the $C_{t-1}$ needs to be passed on as $C_{t}$. Input gate $i$ controls what inputs needs to be added to $C_{t}$. Cell gate $C^{\prime}$ scales the inputs to prepare them to be added to $C_{t}$. Output gate $o$ controls mapping from $C_{t}$ to output $h_{t}$ using inputs $x_{t}$ and $h_{t-1}$.

The LSTM structure is represented mathematically by the following Equations (11). Here the first three equations represents output of the forget gate $f$, the input gate $i$ and the cell update gate $C^{\prime}$ respectively. The fourth equation describes how the inputs of the first three gates are combined to update the cell state. This is represented by the + operator in Fig. 6. The fifth equation is the output $o_{t}$ of the output gate $o$, . And the last equation combines $o_{t}$ and cell state $C_{t}$ to compute output of the cell $h_{t}$. The matrices $W$ are the weights of each gate, and $b$ is the bias term of each gate. The bias and the weights 
will be adjusted during LSTM training.

$$
\begin{aligned}
& f_{t}=\sigma\left(W_{f} \cdot\left[h_{t-1}, X_{t}\right]+b_{f}\right), \\
& i_{t}=\sigma\left(W_{i} \cdot\left[h_{t-1}, X_{t}\right]+b_{i}\right), \\
& \tilde{C}_{t}-\tanh \left(W_{c} \cdot\left[h_{t-1}, X_{t}\right]+b_{C}\right), \\
& C_{t}=f_{t} * C_{t-1}+i_{t} * \tilde{C}_{t}, \\
& o_{t}=\sigma\left(W_{o} \cdot\left[h_{t-1}, X_{t}\right]+b_{o}\right), \\
& h_{t}=o_{t} * \tanh \left(C_{t}\right) .
\end{aligned}
$$

LSTM and its variants like gated recurrent units (GRU, introduced by Cho et al. [41]) have seen huge success in the fields of speech recognition [42], text generation [43], handwriting generation [44], and machine translation [45]. In the next Section 5, we propose a solution that combines LSTM with the cooperative Kalman Filter described earlier to solve the overall problem of tracking a level-curve using a group of robots. 


\section{PROPOSED SOLUTION}

In the Sections 3 and 4 we described how the problem of tracking a level-curve in an unknown field is handled by a group of robots capable of locally measuring the field at discrete intervals. We showed how the measurements can be used with the cooperative Kalman Filter to estimate the field and the field gradient at the center of the formation. Using these field estimates we described the design of the controller used to make the formation center track the desired level-curve. Another controller is used to update the shape of the formation independent of the formation center controller. We also discussed how hessian estimation provides the curvature estimates required by the cooperative Kalman Filter and the formation center controller. This traditional approach involving cooperative Kalman Filter uses the current measurements and previous state of the system to estimate the current state. We propose that if we can use deeper historical data with LSTMs to estimate the current state of the system, we can use fewer robots to measure the field at each step and consequently fewer robots to track the overall level-curve. We also propose a new way to estimate hessian of the field using deeper historical data through LSTMs which allows us to handle more complex fields.

We have three possible model choices to estimate the field and gradient using measurements. 1) The traditional cooperative Kalman Filter as described in Subsection 4.2. 2) A pure LSTM model which takes as input a sequence of measurements (additionally we can also provide other parameters like a sequence of estimated field and gradient values, a sequence of hessian) and estimates the field and gradient. 3) A hybrid model combining LSTM with the cooperative Kalman Filter. There are benefits to each approach. The cooperative Kalman Filter doesn't need data collection or separate training. Additionally, the cooperative Kalman Filter is mathematically proved to converge under certain assumptions [6]. Using LSTM has many benefits. 1) We don't need to explicitly model or design the state equations which needs domain expertise. 2) For complex 
dynamic fields these equations get unwieldy, LSTM would help us here by learning the model from data. 3) As we have a separate training step with LSTM, we can train offline using larger resources, but deploy the trained model using fewer resources. Using a pure LSTM model is ideal to estimate the field and gradient given a sequence of sensor measurements as input. The hybrid model combining LSTM with the cooperative Kalman Filter has some benefits. Here, we use LSTM to predict the state which helps with modeling complex fields and handling a different number of robot sensors, but we still rely on (or take benefit of) the Kalman Filter to correct/tune the LSTM prediction. Next, we describe this hybrid model combining LSTM with the cooperative Kalman Filter in more detail. We compare the experiments between pure LSTM and the hybrid LSTM model in Section 7.

We use the modified LSTM Kalman Filter (LSTM-KF) [35], [36] described by the following Equations (12) - (16). The first two Equations of the cooperative Kalman Filter (5), (6) are replaced by two LSTMs. The LSTMs are given current and previous states and measurement errors as the input. In addition to the current data-point, we provide a number of previous data-points to the model. This is controlled by a hyper-parameter called lag-window. The lag-window can range from 1 (giving only current data) to for example 50 (giving 50 previous data-points). The LSTMs estimate the state and measurement error through the first two equations giving us $s_{k(-)}, P_{k(-)}$. The next three Equations are left the same as in the cooperative Kalman Filter (7), (8), (9), and they use the measurements taken by the robot sensors $p_{k}$ and updated the estimates to give us final state and measurement error $s_{k(+)}$ and $P_{k(+)}$.

$$
\begin{gathered}
s_{k(-)}=\operatorname{LSTM}_{\text {State }}\left(s_{k-1(+)}\right), \\
P_{k(-)}=\operatorname{LSTM} M_{\text {Error }}\left(P_{k-1(+)}\right),
\end{gathered}
$$




$$
\begin{gathered}
K_{k}=P_{k(-)} C_{k}^{T}\left[C_{k} P_{k(-)} C_{k}^{T}+D_{k} U_{k} D_{k}^{T}+R_{k}\right]^{-1}, \\
s_{k(+)}=s_{k(-)}+K_{k}\left(p_{k}-C_{k} s_{k(-)}-D_{k} H_{c, k},\right. \\
P_{k(+)}^{-1}=P_{k(-)}^{-1}+C_{k}^{T}\left[D_{k} U_{k} D_{k}^{T}+R_{k}\right]^{-1} C_{k} .
\end{gathered}
$$

As we have seen in Section 4.1, we need $z_{c}, \nabla z_{c}$ and $H_{c}$ to estimate the updated state using cooperative Kalman Filter. We have also seen in Subsection 4.3 that we need $H_{c}$ to design a controller for the formation center to track the level-curve. The approach described in Subsection 4.5 is an iterative numerical algorithm, which we need to repeat to obtain improved hessian estimates and it is derived for smooth fields. If we use deeper historical data along with LSTM (which is inherently non-linear), we can get more accurate estimates of the hessian, and also expand hessian estimation to more complex curves. So, we use a third LSTM to predict the hessian at the current step. Similar to the other two LSTM models we use a hyper-parameter called lag-window to control how much of the history is presented to the LSTM to predict the current hessian. We use the traditional hessian estimation method described in Subsection 4.5 to decide the inputs for the LSTM to predict the hessian. Specifically, our 3rd LSTM's inputs are comprised of formation center $r_{c}$, field $z_{c}$, gradient $\nabla z_{c}$, and previous hessian value at the center $H_{c}^{P}$ ), robot locations $r_{i}$, and measurements $z_{i}$. This is denoted by:

$$
H_{c, k}=\operatorname{LSTM}\left(r_{c}, z_{c}, \nabla z_{c}, r_{i}, z_{i}, H_{c, k-1}\right) .
$$


This predicted hessian $H_{c, k}$ is used in the Kalman Filter Equation (15) and formation center control law (10) described earlier. The flowchart of the complete proposed model is captured in Fig. 7.

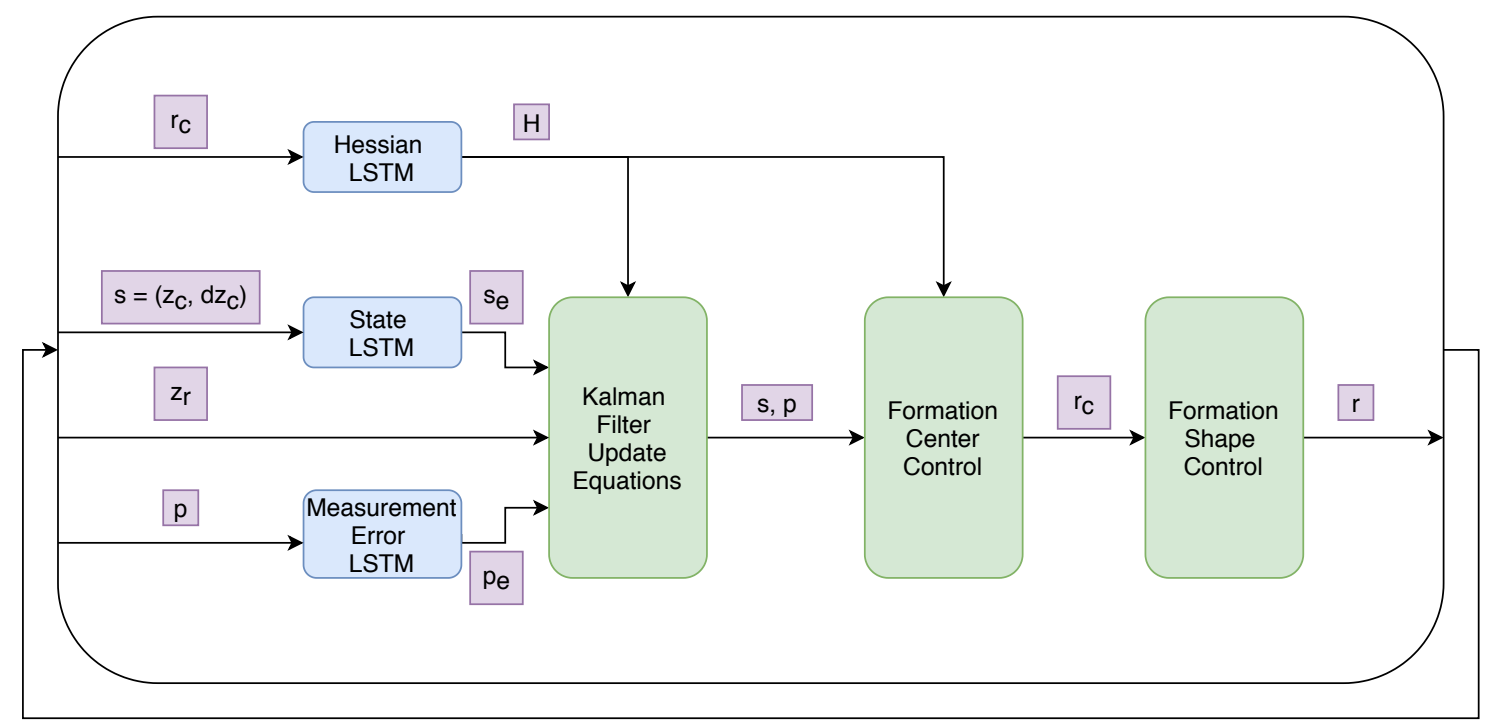

Fig. 7: The proposed motion control flow using estimates generated through LSTM-KF. $r$ represents sensor locations and $z_{r}$ represents sensor measurements. $z_{c}$ and $d z_{c}$ represents field and gradient at the center $r_{c}$. $s$ represents the state of the system. $p$ is the error co-variance matrix for state estimation. $H$ represents estimated hessian of the field. $s$ and $p$ are passed in from the previous time-step $k-1$, the LSTMs generate predicted estimates $s_{e}$ and $p_{e}$. The cooperative Kalman Filter uses $z_{r}$ to update the predictions and provide $s$ and $p$ for current time-step $k$. Formation center and formation shape controllers use these estimates to calculate updated $r_{c}$ and $r$ for time-step $k$.

We have considered multiple LSTM structures while evaluating the accuracy of the results. In the figure below, we provide an overview of some of the models which are trained to predict field and field gradients, i.e. $z_{c}, \nabla_{x} z_{c}, \nabla_{y} z_{c}$. In combination, these three values make up the state of the system. The first model (Fig. 8) consists of a single LSTM layer containing 40 neuron units followed by a dense layer to combine the LSTM results and predict three values. This model captures the LSTM-KF described in Equations (12), (13), the model only uses historical states and no actual measurements to predict the future state. 


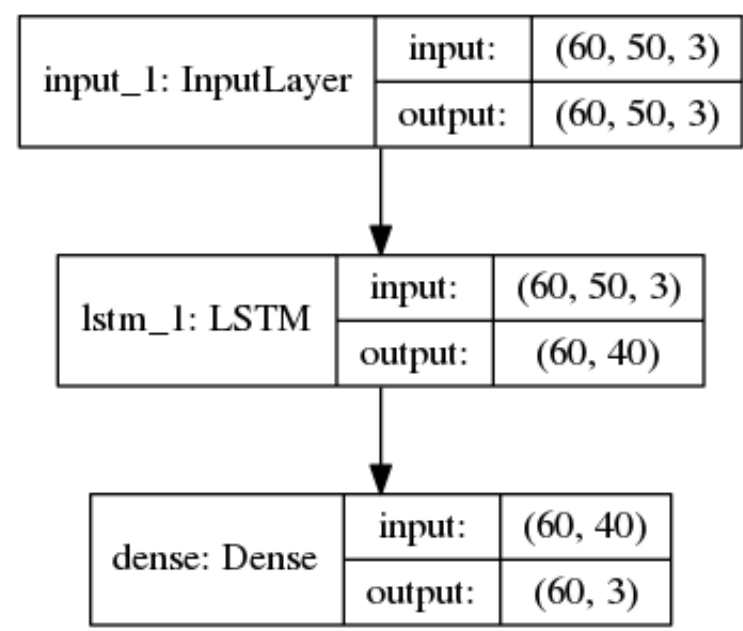

Fig. 8: Single LSTM layer model containing 40 LSTM units. 60 represents the batch size, 50 is the lag window, i.e. LSTM is given $s_{k-50}, s_{k-49}, . ., s_{k-1}$ as inputs to predict $s_{k}$. This LSTM has 3 features $z_{c}, \nabla_{x} z_{c}, \nabla_{y} z_{c}$.

The next model (Fig. 9) contains two LSTMs in parallel. The first LSTM uses the historical three state values $z_{c}, \nabla_{x} z_{c}, \nabla_{y} z_{c}$ as input. The second LSTM uses formation center $r_{c}$, sensor measurements $z_{r}$ and hessian $H_{c}$ values to predict the state. Finally, the two predictions are combined using a dense layer. This was an attempt to use robot measurements and incorporate the fourth equation of the cooperative Kalman Filter (Equation (8)) inside the LSTM model. This model uses the sequence of measurements directly in the LSTM, and so it can be used as a pure LSTM model without having the cooperative Kalman Filter to update the predictions. 


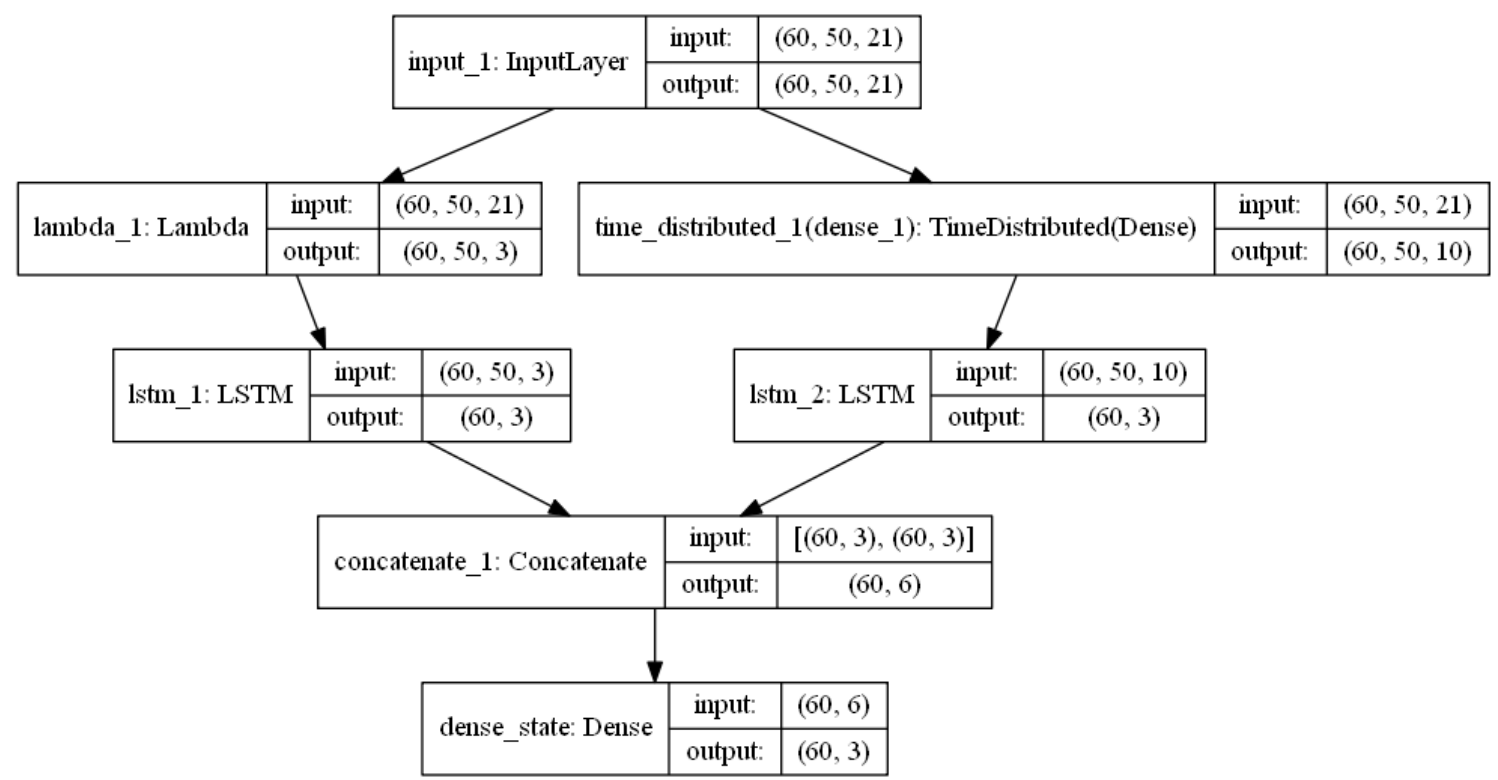

Fig. 9: Independent LSTMs using state and measurements. The left branch shows LSTM similar to Fig. 8 that uses $s_{k-i}$ to predict state estimate $s_{k}$. The right branch shows LSTM that uses 10 features ( 4 features of hessian $H, 2$ features containing $r_{c}, 4$ features of $z_{r}$ ) to predict $s_{k}$ - this mimics cooperative Kalman Filter's update equation (Equation. (8)). There are 21 total features in the dataset of which 3 and 10 are selected for the left and right branch respectively. The final dense layer combines the outputs of two LSTMs to estimate final $s_{k}$. Batch size is 60, lag window is 50, and the LSTMs contain 3 unit cells each.

The third model (Fig. 10) doesn't use LSTM, it uses fully connected dense layers. We collect field value at the center and robot measurements $z_{c}$ and $z_{r}$ (i.e. 5 features) for the previous three steps (i.e. lag-window $=3$ ). Next, we flatten the 5 features over 3 time-steps to get 15 features. These 15 features are passed to the fully connected layer which combines them using trained weights and predicts the current state. The intuition behind this model is that we hope to train the weights of the fully connected layer, which will result in a set of non-linear equations that can predict the current state using the given data from previous time-steps as input. 


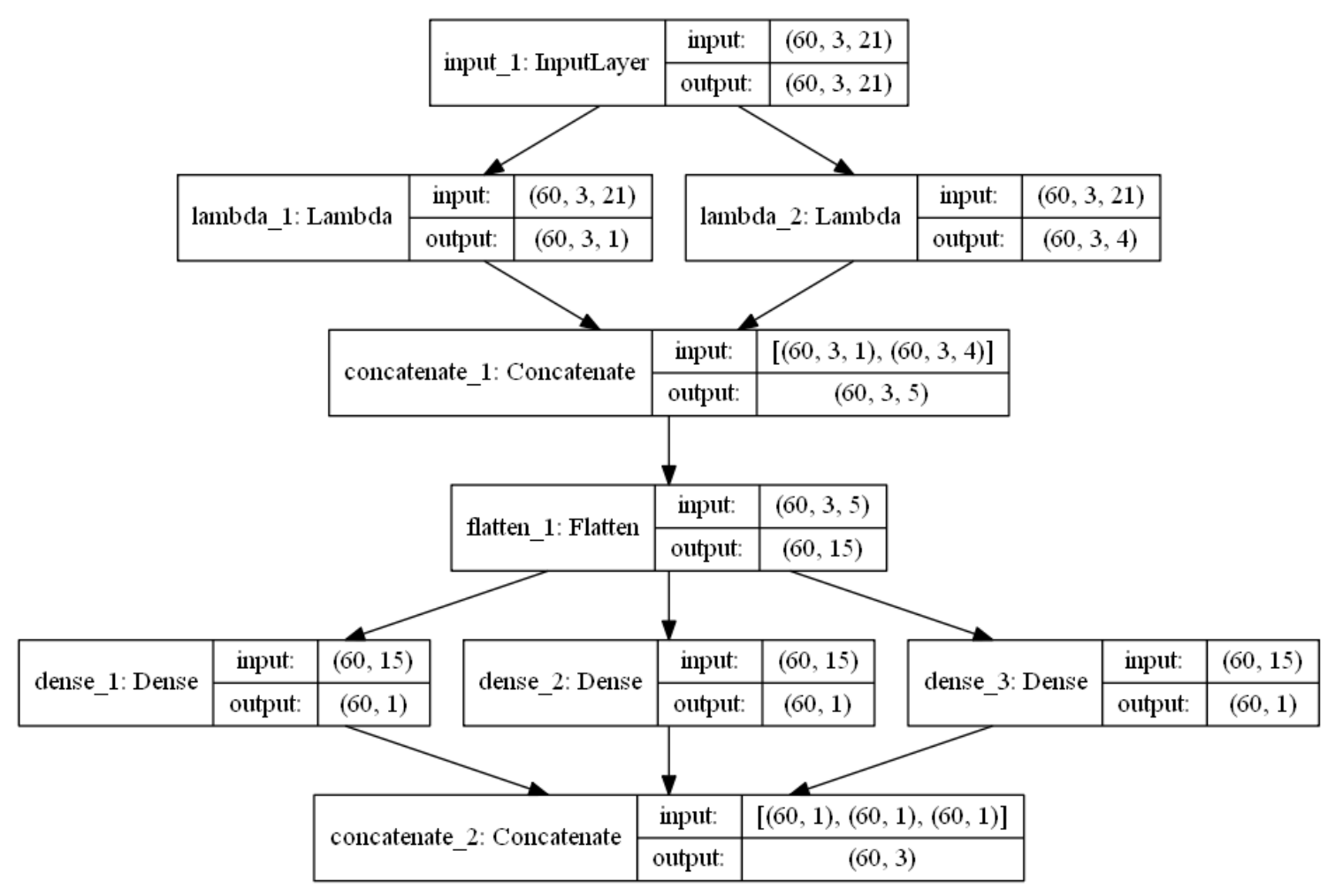

Fig. 10: Combine field and measurements using a fully connected layer. First we collect 5 features ( 1 feature as $z_{c}$, and 4 measurements $z_{c}$ ). We collect 5 features over 3 time-steps and flatten to form a vector of 15 values. We apply a fully connected dense layer to combine the 15 values to predict the state $s_{e}$.

The last model (Fig. 11) contains three LSTMs running in parallel and independent of each other - one for each of the state variables $z_{c}, \nabla_{x} z_{c}, \nabla_{y} z_{c}$. The state is predicted using previous state values $z_{c, k-i}$. The gradients are predicted for $\mathrm{X}$ and $\mathrm{Y}$ direction using historical gradients $\left(\nabla_{x} z_{c, k-i}\right.$ and $\nabla_{y} z_{c, k-i}$ respectively) and historical formation centers $r_{c, k-i}$. Here i ranges from 1 to $\left(l_{a g} \_\right.$window -1$)$. This model was chosen to account for the fact that when the formation center takes a sharp turn on the level-curve we need to tune field gradients considerably, as we will notice in the gradient plots in the Section 7. With independent LSTMs for the gradients, the loss of each LSTM can be reduced to make each gradient prediction more accurate, rather than increasing the overall accuracy of combined field and gradient prediction. 


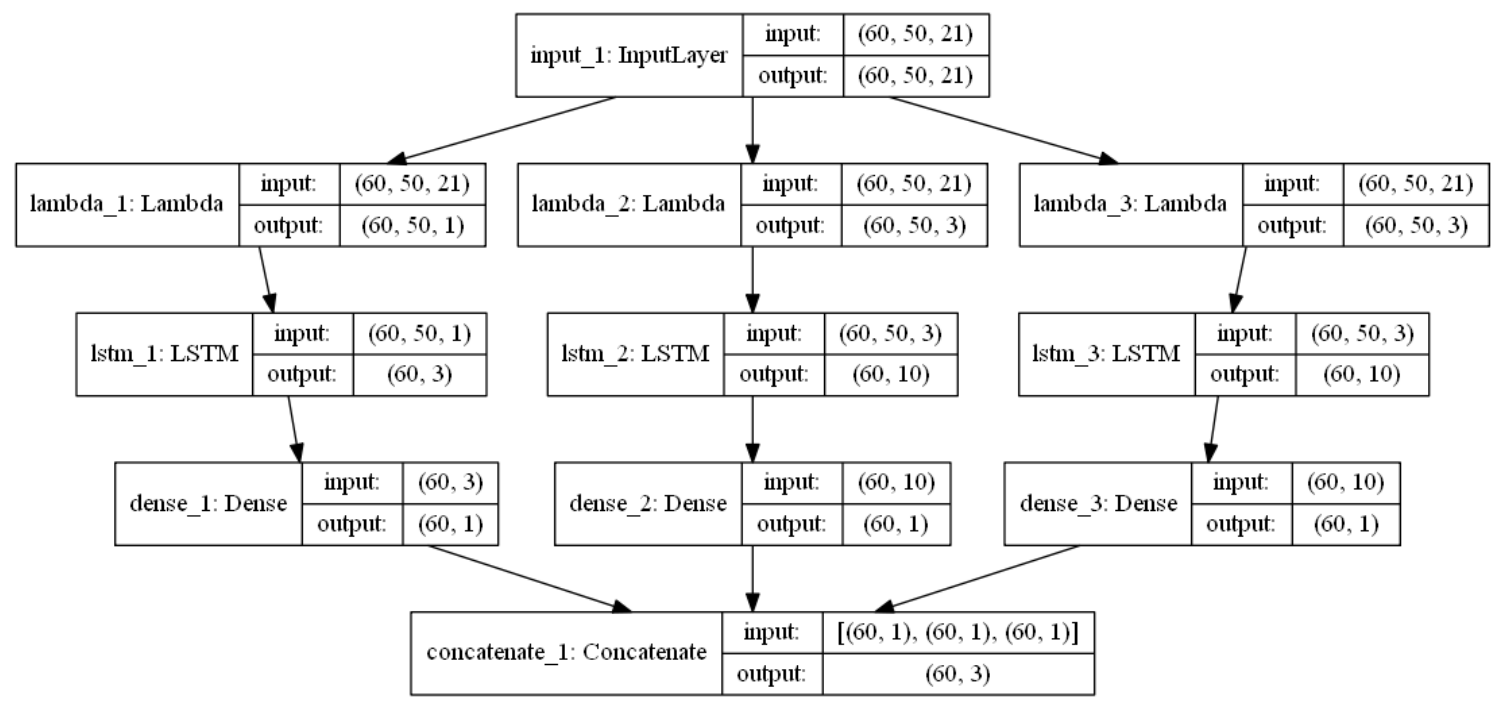

Fig. 11: Field and gradients independent LSTMs. This is similar to simple one layer LSTM model (Fig. 8) except we have 3 independent LSTMs for each of the state feature $z_{c}, \nabla_{x} z_{c}, \nabla_{y} z_{c}$

In the Section 6, we discuss in more detail how we collected the data using traditional cooperative Kalman Filter enabled motion control strategy (Subsection 6.2.1). We then describe how we trained the various LSTM models on the collected data, including steps of data preparation (Subsection 6.2.2). And finally, we describe the framework used to test the model in unseen fields (Subsection 6.2.3). We also describe in detail the error metric we observed to compare various hyperparameters. We tune various hyperparameters like batch-size, lag-window, number of LSTM, and dense layer neurons in each layer, amount of data used to train the model and variations of the four models we described in this section. We perform a manual grid-search over these parameters and select the best performing model. 


\section{IMPLEMENTATION DETAILS}

We use the proposed solution described in Section 5 and design an experiment to first train the LSTM models, and then test it in unknown fields. We compare these results with the results we get using existing technology. We first provide an overview of the experiment, and then we describe the software used to collect data from the experiments and train the LSTM models for the state and measurement errors. Lastly, we describe how we train the hessian estimation model.

\subsection{Overview}

We need data for training the three LSTM neural networks. For this reason, we first start with the existing cooperative Kalman Filter and generate data for a smooth 2D field. For the equations described by Zhang and Leonard [6], we need 4 robots for a static formation and at the minimum, we need 2 robots. If we use 2 robot formation, we need to constantly oscillate the robots around the formation center. This oscillation is necessary so that we can compute the gradients in both $\mathrm{X}$ and $\mathrm{Y}$ direction. Without the oscillations we end up with a singular matrix in the Kalman Filter. Using such a formation of 2 robots also leads to a slight loss in accuracy in comparison to the 4 robots static formation. Additionally, Wu et al. [20] proved that we need at least 6 robots in a 3D field to sufficiently measure the required data and for the Kalman Filter to converge. 
In this project for 2D fields, we consider 4 robot formation to simulate the traditional Kalman Filter and to collect the training data. One such training field is shown in Fig. 12. The figure shows an irregular field obtained using a $4^{\text {th }}$ order 2D polynomial. To collect data for training the LSTMs, given a starting point for the 4 robots, we set the desired field value to 144 . At each point, the sensors measure field values. The previous state and measurements are passed to the cooperative Kalman Filter (Equations (5) - (9)) to estimate the current state. The estimated state is passed to the formation center (Subsection 4.3) and formation shape (Subsection 4.4) controllers to update the trajectory to track desired the level-curve.

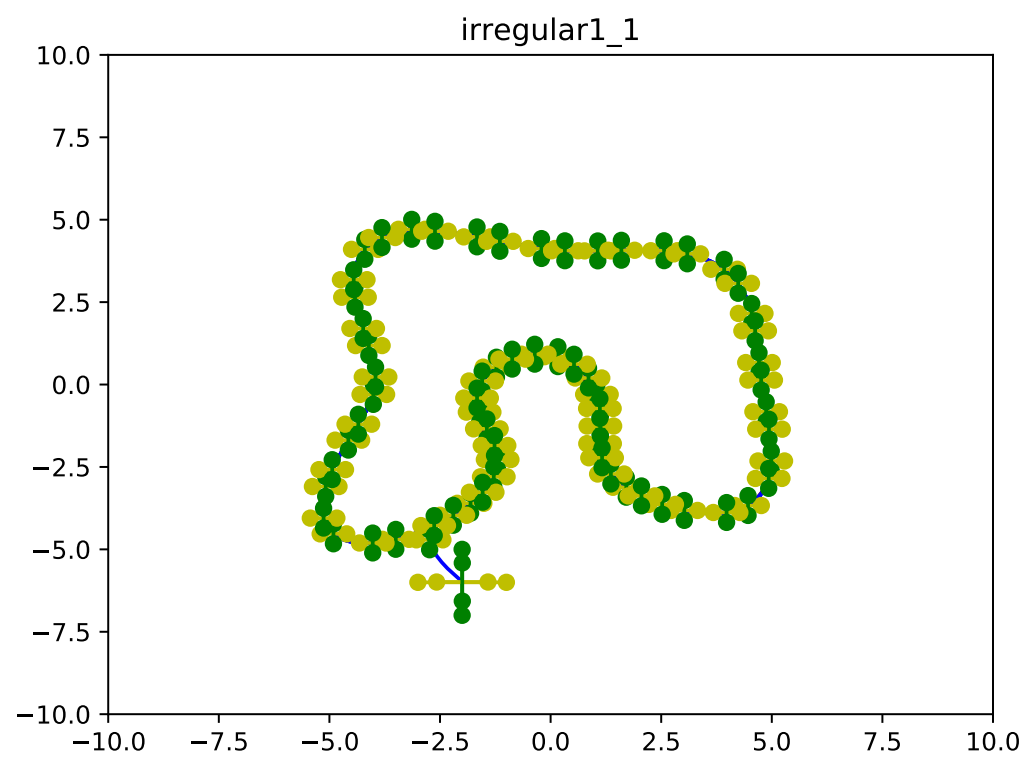

Fig. 12: Irregular field tracked by 4 robots. The figure shows one of the training levelcurve shapes. The sensor formation starts with the formation center at $(-2,-6)$. Green dots represent sensors $r_{1}$ and $r_{2}$, the green line is an imaginary line connecting them and passing through $r_{c}$. Similarly yellow represents $r_{3}$ and $r_{4}$ sensors. The trajectory traced by the formation center is shown in blue (minimally visible).

Once we have the training data, we feed the current and a number of historical states $s_{k-i(+)}$ and errors $P_{k-i(+)}$ to the LSTM models as described in the first two Equations (12), (13) of the LSTM-KF model. The LSTM models predict the next state $s_{k(-)}$ and 
error $P_{k(-)}$. The actual next state and error collected as data earlier $\left(s_{k(+)}, P_{k(+)}\right)$ are given as feedback to the LSTM to back-propagate the weights and learn the cooperative filter. The LSTMs are trained to minimize the root mean square error while predicting the state matrix and the error matrix.

Finally, to test the system (Fig. 7), we use the LSTM-KF model equations which are described next. Given the current and historical data, the LSTM models (Equations (12), (13)) predict the estimated current state $s_{k(-)}$ and the error matrix $P_{k(-)}$. Then the robot measurements $p_{k}$ update the state $s_{k(+)}$ and error matrix $P_{k(+)}$ - correcting them with actual field values using Equations (15), (16). These final values are provided to subsequent controllers to correct the formation-center's trajectory (Equation (10)) and to correct the shape of the formation (Subsection 4.4) for optimal data collection. The algorithm 1 describes the overall algorithm. 


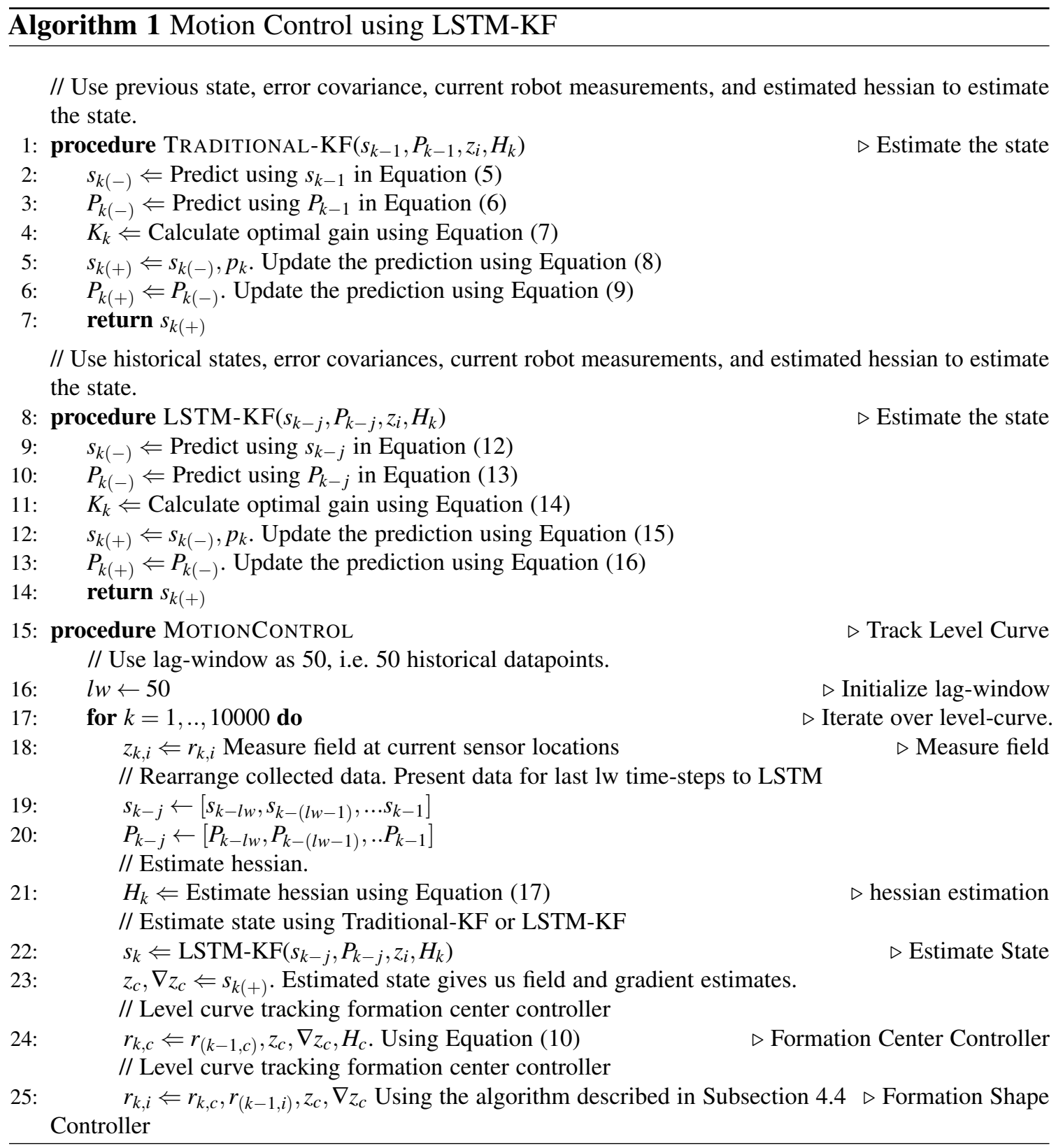




\subsection{Motion Control}

We use python and associated libraries like Sklearn, Keras for our simulations. We first implement motion control using the cooperative Kalman Filter as described in [6]. The motion control has four main procedures (specifically hessian estimation, cooperative Kalman Filter, formation center controller, formation shape controller) which are repeatedly called at each point along the trajectory.

Next, we describe the three main phases in which the whole program is run: Data Collection, Training model, Testing.

\subsubsection{Data Collection}

First, we measure field value at the location of the robots $z_{r}$ using the polynomial function representing the field $f$ and the $x, y$ coordinates of individual robots $r_{i, k-1}$, this mimics real sensors in simulation. Next, we calculate the hessian at the center of the formation. During training, we use the true second derivative of the polynomial function $\nabla^{2} f$ to calculate the hessian at the formation center $H_{c}$.

Next, we call the kalmanFilter procedure (Algorithm line 1) which consists of the five equations that make up the Kalman Filter (Equations (5) - (9)). This provides the updated state of the robot system $s_{k(+)}$ : this consists of field $z_{c}$ and gradient values $\nabla z_{c}$ at the formation center. The cooperative Kalman Filter also returns the error covariance matrix $P_{k(+)}$, which we hold on to, to it back to the Kalman Filter for the next iteration when we are on the next point along the trajectory.

We pass these field and field gradient values to the formationCenter function (Algorithm line 24). The function uses the previous formation center location $r_{c, k-1}$ and the estimated field gradients to calculate the updated formation center $r_{c, k}$ which tracks the desired field value $z_{\text {desired }}$. After this we call formationControl function (Algorithm line 24) which updates the formation shape of the four robots, i.e. individual robot's 
location $r_{i, k}$. For these two functions, we use the controllers described in Subsections 4.3 and 4.4 .

Below we list the inputs and outputs to each of the three main functions Kalman Filter, formation-center controller, formation-control controller.

$$
\begin{gathered}
z_{c}, \nabla z_{c}, p=\text { kalmanFilter }\left(z_{c}, \nabla z_{c}, r, z_{r}, r_{c}, r_{c, o l d}, p, H_{c}, \text { model }\right) \\
r_{c}, x_{2}, y_{2}=\text { formationCenter }\left(r_{c}, z_{c}, \nabla z_{c}, H_{c}, x_{2}, y_{2}, \text { mu }_{f}, z_{\text {desired }}\right) \\
r, q, d q, u_{r}, \text { vel }_{q}=\text { formationControl }\left(r_{c}, r, q, d q, u_{r}, \text { vel }_{q}\right)
\end{gathered}
$$

Here, $z$ is the field value, $d z$ is the field gradient value (same as $\nabla z_{c}$ ), $r$ is the robot locations, $z_{r}$ is sensor measurements, $c$ stands for formation center, $p$ is the error matrix. The rest of the variables like $x_{2}, y_{2}, m u_{f}, q, d q, u_{r}, v e l_{q}$ are necessary for the controllers and they maintain the state of the controller, but they are not notable for our purposes. Some of those variables are like $x_{2}, y_{2}$ which represents the heading of the formation, $q, d q, v e l_{q}$ denoting the Jacobian representation of the formation shape. For LSTM-KF the trained model is also passed in as an input to the kalmanFilter to predict the new state and error matrix.

Once we have these new robot locations we go back to the first step of calculating field values at the robot locations $z_{i, k}$ and repeat all the steps. Thus, we end up with a set of points traced by the robots after say 10000 iterations. This set of points form the level-curve along the chosen field at the desired field value $z_{\text {desired }}$.

Thus we first simulate tracking the level-curve with autonomous MWSN, in which we use cooperative Kalman Filter to estimate the state and motion controllers to decide the trajectory. Throughout this simulation at each of the 10000 time-step $k$, we save the field values $z_{c, k}$, gradients $\nabla z_{c, k}$, error matrix $P_{k}$, hessian $H_{k}$, sensor measurements $z_{i, k}$, robot locations $r_{i, k}$ and formation center $r_{c, k}$. We store this in a Python dataframe on disk. Next, 
we repeat this experiment for other field shapes. Thus we end up with multiple dataframes - one for each shape. This series of dataframes is stored in a pickled file on disk. This gives us a sequence of data to train LSTMs. We consider a variety of shapes like circles of different polynomial degrees, ellipses with different coefficients, a few $4^{\text {th }}$ order $2 \mathrm{D}$ irregular polynomial shapes with varying coefficients. For example, one of the $4^{\text {th }}$ order polynomial shapes is:

$$
\left(x^{2}+2 y-12\right)^{2}+\left(K * x+y^{2}-17\right)^{2}=400,
$$

where 400 is the desired field value $z_{\text {desired }} K$ is the coefficient we vary to generate more shapes.

\subsubsection{Training Model}

The data is collected through simulation and not observed from real world measurements. We have total control in the simulation over which variables are collected. So, the data doesn't have to be cleaned up for missing values, removing anomalies, resampling, etc. We perform three main steps in data preparation for LSTMs. First, we flatten all the various vectors and matrices like state $s_{e}$, hessian $H_{c}$, formation center $r_{c}$, robot locations $r_{i}$, sensor measurements $z_{r}$, error covarience matrix $P$ into a single vector of 21 features for each time-step. Therefore, we get a $10000 \times 21$ matrix corresponding to the 10000 timesteps for a single level-curve shape. Second, we scale each feature using MinMaxScaler down to $[-1,+1]$ range. Finally, we reframe the data for supervised learning in the form of data $X$ and labels $Y$. This reframing procedure is described next. For LSTMs, we need to decide on a hyper-parameter called lag-window. Lag-window is the amount of historical data we show to the LSTM to predict the current state. In the analysis section, we provide some insight into how we can get a range for this parameter in order to tune it. For example, if we choose lag-window to be 50, for the label at time-step $\mathrm{k}\left(y_{k}\right)$ we need to provide data from 50 previous time-steps 
$\left(\left[x_{k-50}, x_{k-49}, . ., x_{k-1}\right]\right)$. We re-organize the data in this fashion using a sliding window method. We end up with data $X$ and labels $Y$ matrices of the following format for training the state estimation LSTM:

$$
\begin{gathered}
X=\left[\begin{array}{cccc}
{\left[z_{c, 0}, \nabla_{x} z_{c, 0}, \nabla_{y} z_{c, 0}\right]} & {\left[z_{c, 1}, \nabla_{x} z_{c, 1}, \nabla_{y} z_{c, 1}\right]} & . . & {\left[z_{c, 49}, \nabla_{x} z_{c, 49}, \nabla_{y} z_{c, 49}\right]} \\
{\left[z_{c, 1}, \nabla_{x} z_{c, 1}, \nabla_{y} z_{c, 1}\right]} & {\left[z_{c, 2}, \nabla_{x} z_{c, 2}, \nabla_{y} z_{c, 2}\right]} & . . & {\left[z_{c, 50}, \nabla_{x} z_{c, 50}, \nabla_{y} z_{c, 50}\right]} \\
\cdot & \cdot & \cdot & \cdot \\
\cdot & \cdot & \cdot
\end{array}\right], \\
Y=\left[\begin{array}{ccc}
z_{c, 50} & \nabla_{x} z_{c, 50} & \nabla_{y} z_{c, 50} \\
z_{c, 51} & \nabla_{x} z_{c, 51} & \nabla_{y} z_{c, 51} \\
\cdot & \cdot & \cdot \\
\cdot & \cdot & \cdot
\end{array}\right],
\end{gathered}
$$

where $X$ is of the shape $(9950 \times 50 \times 3)$ and $Y$ is of the shape $(9950 \times 3)$. For training the error co-variance LSTM, in which each error co-variance is a $(3 \times 3)$ matrix, we Similarly we obtain $X$ and $Y$ matrices for error co-variance of the shape $(9950 \times 50 \times 9)$ and $(9950 \times 9)$ respectively.

The entire set of level-curves, each having $X$ and $Y$ matrices as described above, is split into three parts. One set is used to train the model, another for model validation, and the last set of level-curves are withheld from the model during the training. The last set of level-curves will be used during the testing phase. Now we train the state and error LSTM models using the prepared training data. We use standard methods like early stopping, model checkpoints to save only the best model. We use model validation to improve the generalization of the trained model. We train for 200 epochs with early-stopping patience of 40 epochs, so if the validation-loss doesn't improve for 40 consecutive epochs we stop training further on that shape. Here, an epoch means going once over all the 9950 data points for a shape. This will be repeated 200 (epoch) times to improve the learned weights. The LSTM is trained using the Keras model.fit method with the objective to reduce mean squared error on validation loss, and we use the Adam optimizer for gradient descent. 
There are a few unique points we need to consider while training LSTMs. First is we can't shuffle the data because the pattern of the data matters. So, we train through each shape and used the learned model weights (instead of random weights) when starting with the next shape. This means by the end each shape, which is part of the training data-set, has contributed to the learned weights. LSTM also maintains an internal state across all neurons, we flush this internal state after each epoch, and when we move from one shape to another. Second, for each step of LSTM training, we need to provide the data $X$ in the (batch-size, lag-window, num-features) format and labels $Y$ in (batch-size, num-features) format. Batch-size is selected to be 60 after manual grid-search to speed up the training but still derive a well-trained model. In order to account for the batch-size, the matrix $X$ and $Y$ described above need to be reorganized to be given 60 rows at a time.

\subsubsection{Testing}

Finally, for the testing phase, we use LSTM-KF as described through Equations (12) (16) . We replace the first two equations of Kalman Filter with LSTM predictions from the

trained model. This is described in the Algorithm line 8. The rest of the flow remains the same as when we collected the data (Subsection 6.2.1). If the lag-window hyperparameter is 50 , during the testing phase, we remember the previous 50 feature values. For the state LSTM at any time step, 50 historical state values are passed in to predict the current state, and similarly for the error covariance LSTM to predict $P_{k}$. These state and error covariance predictions are passed into the cooperative Kalman Filter's update equations to generate the updated state and error matrix. These matrices are cached for use with the next 50 time-steps. The state estimate is then passed on to the formation center controller which updates $r_{c}$ to track the level-curve with the field value of $z_{\text {desired }}$. This updated formation center and gradient estimates are passed to the formation shape controller to update the individual sensor locations. Additionally, the third LSTM predicts the hessian at the formation center $H_{c}$, which is used by the cooperative Kalman Filter's update 
equation and the motion controllers. We iterate these steps 10000 times and plot the trajectory of the formation to note whether the formation tracks the desired level-curve.

We evaluate the results by manual inspection of the trajectory. Additionally, we define an error metric to evaluate the performance of the trained LSTM-KF model in regards to the overall motion control objective to track the level-curve. We plot the ideal field and gradient values over 10000 iterations and compare that with the plot of estimated field and gradient values by the LSTM-KF model. One such plot is shown in Fig 16. We calculate the numerical root mean square error (RMSE) summing up the between actual and real values over the entire trajectory. We use these three RMSE errors one for each of the field and the gradient in each direction to compare the performance of the trained LSTM-KF model.

One sample error metric result of an experiment is shown in Fig. 13. This error metric is used internally to perform experiments quickly while varying hyper-parameters and we have found it to be useful, but it is not our final deciding factor. We also depend on manual inspection of the plot of the trajectory for final acceptance. We perform many experiments with different variations such as training different LSTM models (as described in Section 5), increasing or decreasing the lag-window, increasing the number of LSTM units, down-sampling the training data during data-preparation to focus on long-term trends, varying the size of dense layers and even varying controller parameters such as $K_{4}$ in Eq. 10. 
Tracing shape for circle_1.0

RMSE for shape z: 0.7199219248015771 RMSE, Max: 40.0, Min: 16.017513206432415

RMSE for shape dz_x: 2.468482713213803 RMSE, Max: 8.005944736210374, Min: -8.005944583589578

RMSE for shape dz_y: 5.046551564089801 RMSE, Max: 8.005944795128176, Min: -12.0

Tracing shape for elipse_1.0

RMSE for shape z: 0.8939384266212096 RMSE, Max: 66.0, Min: 16.003752427907266

RMSE for shape dz_x: 2.7581238811421342 RMSE, Max: 8.33920170052272, Min: -8.339201157168375

RMSE for shape dz_y: 7.740948205867986 RMSE, Max: 14.441175198936453, Min: -28.0

Tracing shape for irregular2_8.0

RMSE for shape z: 20.565989960774644 RMSE, Max: 421.93723067110335, Min: 400.25836420768167

RMSE for shape dz_x: 405.9853342808444 RMSE, Max: 449.36385499269335, Min: -629.9315482122752

RMSE for shape dz_y: 464.42607051843163 RMSE, Max: 521.2631901157159, Min: -723.0805693297829

Fig. 13: Error metric for evaluating the trained model. The figure shows error over three test level-curves: circle, ellipse and a irregular shape. For each shape we calculate three RMSE errors: for field $z$, and for gradients in the X and Y direction $d z_{x}$ and $d z_{y}$ respectively. We also specify the min and max of each variable to compare the RMSE error against the range of these variables.

While testing we try two important experiments, first we test on shapes unseen during training and validation. Second, we also try changing the number of robots $N$. We trained using four robots, but we test using two robots. Succeeding in the first experiment shows that we can handle unknown shapes, i.e. the trained LSTM model generalizes well. The second experiment shows that we can get away with using a lesser number of robots during testing, even though we used a higher number of robots during training to collect more accurate data.

\subsection{Hessian Estimation}

We train the hessian LSTM separately. For collecting data for hessian estimation we bypass the Kalman Filter and use true values of the field, gradient, and hessian values at each time step. We collect all the required variables in a dataframe for each shape and we end up with a similar series of dataframes for a set of shapes. We discussed the required variables and the reasons for selecting them for the hessian LSTM in the proposed 
solution Equation (17). The following variables are given as the input to the LSTM: $r_{c}, z_{c}, \nabla z_{c}, r, z_{r}, H_{c, k-1}$

Next, we train a third LSTM network on the data. The network has an LSTM layer of 22 units followed by a dense layer of 4 units to output the four hessian values. Similar to the motion control LSTM, we use lag-window as a hyper-parameter, and train for 200 epochs with early stopping with the patience of 40 epochs. This trained LSTM is then used to predict the hessian values and the estimates are then used with the LSTM-KF. This hessian values are also necessary for the formation-center controller. 


\section{RESULTS}

Here we present some of the results generated by trained LSTM models. First, we discuss 4 robot formations in which we see that the LSTM model can generalize outside of shapes seen during training. After that we see a two robot oscillating formation for the circle shape, in which the model was trained using a 4 robot formation but tested on 2 robot formation.

Through experimentation we observe that out of the four models proposed in Section 5, the first model (Fig. 8) works the best. It consists of a single LSTM layer containing 40 neurons followed by a dense layer. We use the same configuration for all the three LSTMs. We observe that the state LSTM is the most important, and any inaccuracy in state LSTM has a large effect on the results. We also tried a stacked LSTM model which consists of back to back LSTMs followed by a dense layer. The benefit of such a structure is that it would be able to learn both the short and the long term patterns. However, we don't see much improvement in using the stacked LSTM. We have tried pure LSTM models very similar to model shown in Fig. 9, but we observe that without the cooperative Kalman Filter's state-update equation (15). The trajectory taken by the autonomous MWSN diverges from the desired level-curve after some time-steps when the level-curve takes a turn. Thus, we focus mainly on tuning the hybrid LSTM-KF model for best performance. 
The trajectory traced by 4 robot formation robots using estimates produced by the traditional cooperative Kalman Filter is shown in Fig. 14. In this figure, the violet line shows the desired level-curve, and the blue line shows the trajectory taken by the robots. The trajectory traced by 4 robot formation robots using the field estimates predicted the trained LSTM model is shown in Fig. 15. We see that the robots don't trace the curve well for some of the concave curves. We see that for these shapes the traditional cooperative Kalman Filter performance is better than LSTM-KF for the field and gradient estimates. However, the plots show a proof of concept that LSTM-KF can be used for estimating the field characteristics for level-curve tracking problems. The benefit of LSTM-KF is that it doesn't require explicitly deriving or modeling the state system, so for more complex scenarios such as dynamic time-varying fields the LSTM-KF method is more practical. We further show that LSTM-KF can be trained using larger resources and deployed with limited resources. 

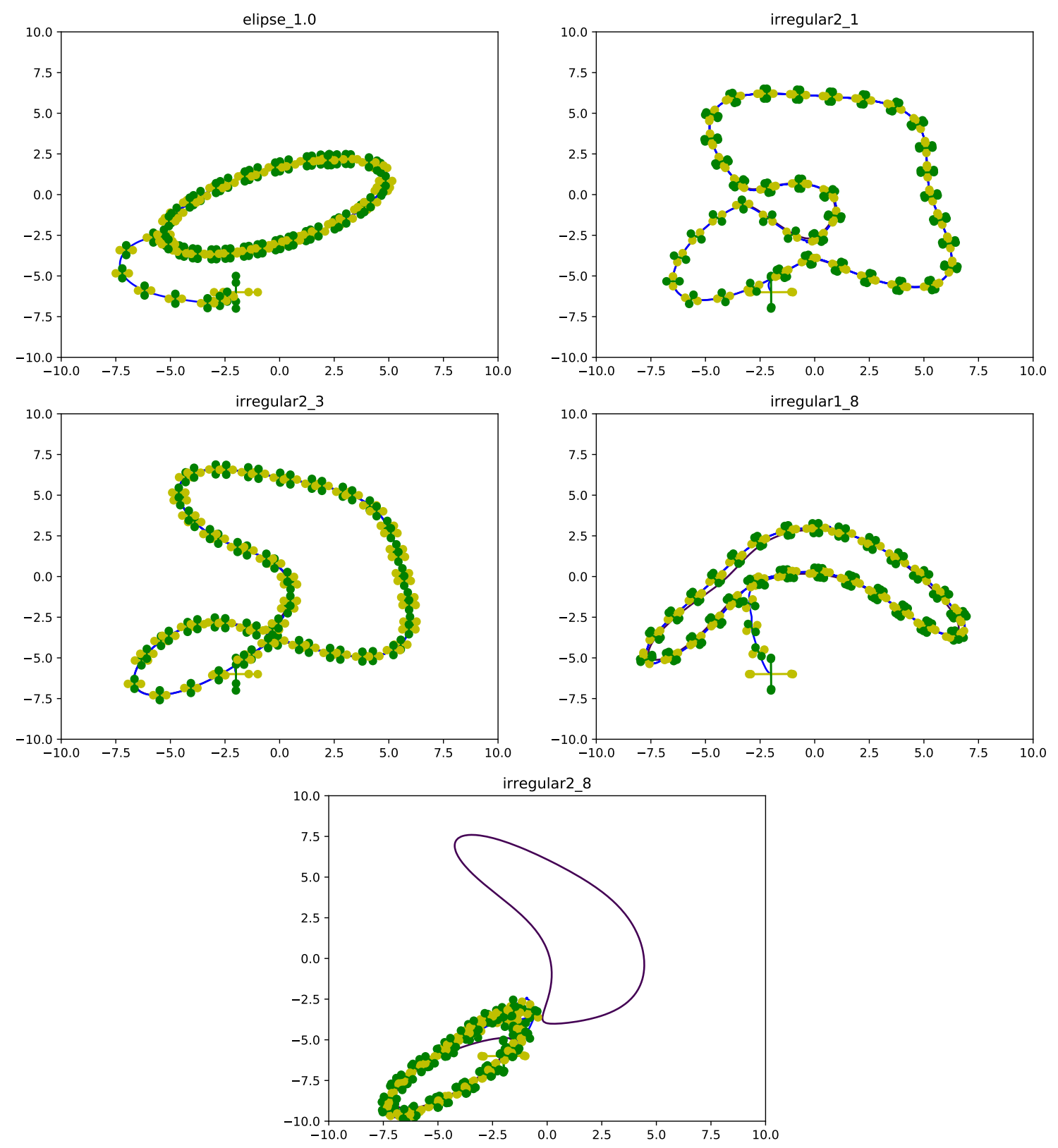

Fig. 14: Field traced by robots using traditional Kalman Filter. The figures show 4 robots formation, green representing robots $r_{1}$ and $r_{2}$, yellow representing robots $r_{3}$ and $r_{4}$. The desired level-curve is shown in violet. The formation starts with the center $r_{c}$ at $(-2,-6)$. The blue level-curve is the actual trajectory followed by the simulated sensor network. 

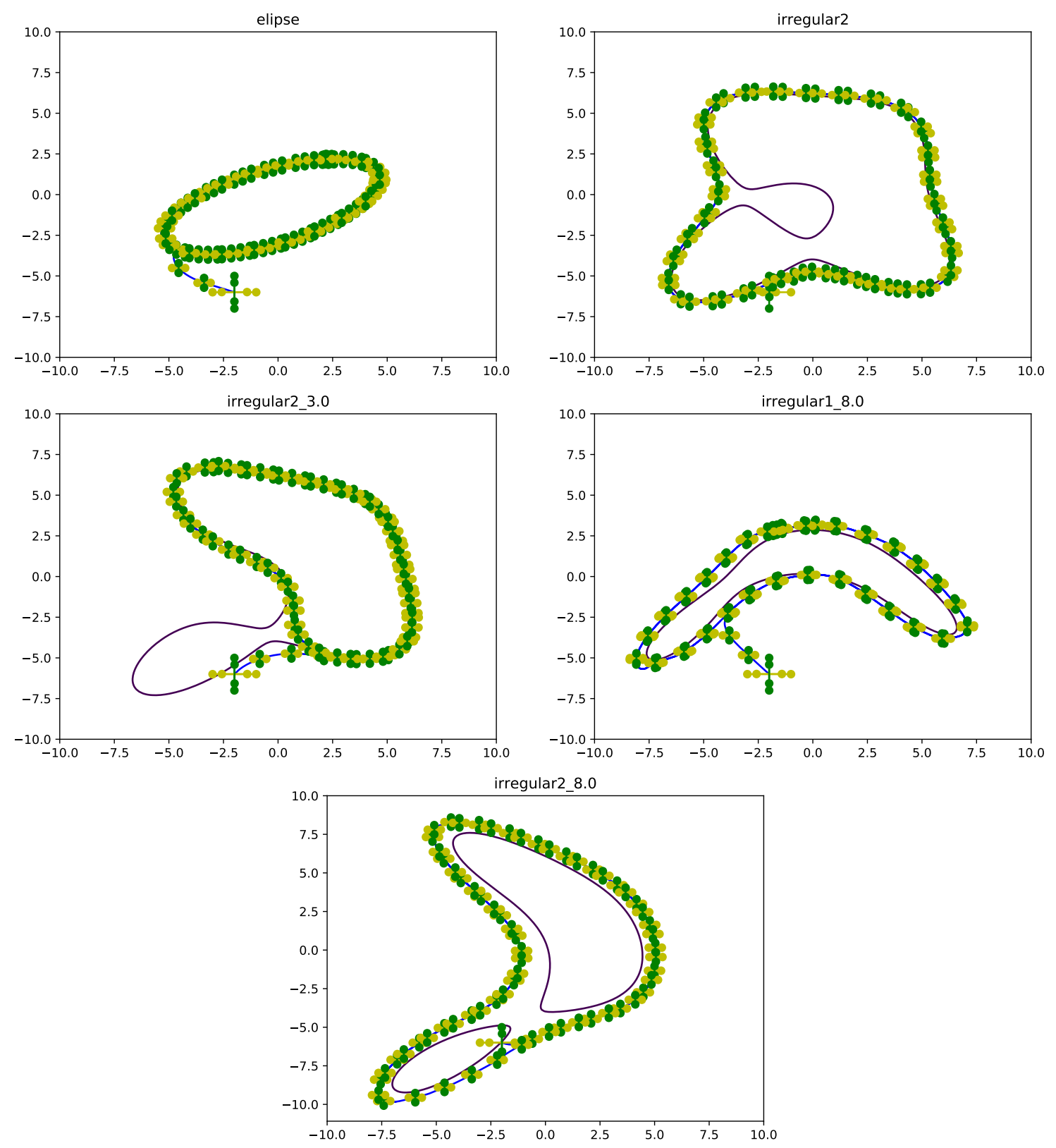

Fig. 15: Field traced by robots using the predictions by LSTM-KF. The figures show 4 robots formation, green representing robots $r_{1}$ and $r_{2}$, yellow representing robots $r_{3}$ and $r_{4}$. The desired level-curve is shown in violet. The formation starts with the center $r_{c}$ at $(-2,-6)$. The blue level-curve is the actual trajectory followed by the simulated sensor network. We use the proposed motion control strategy (Fig. 7) for simulation which uses the LSTM-KF design. 
We also plot in Fig. 16 (for the irregular1_8 shape in Fig. 15) the field and field gradients to show the error between actual shape and modeled trajectory. Here red plot shows the values we get if we follow the actual level-curve which is obtained using traditional Kalman Filter, green are the values we get using LSTM. The error (vertically) at each point (X-axis) determines the root mean square error. We try to reduce this root mean square error when we train the LSTM model.

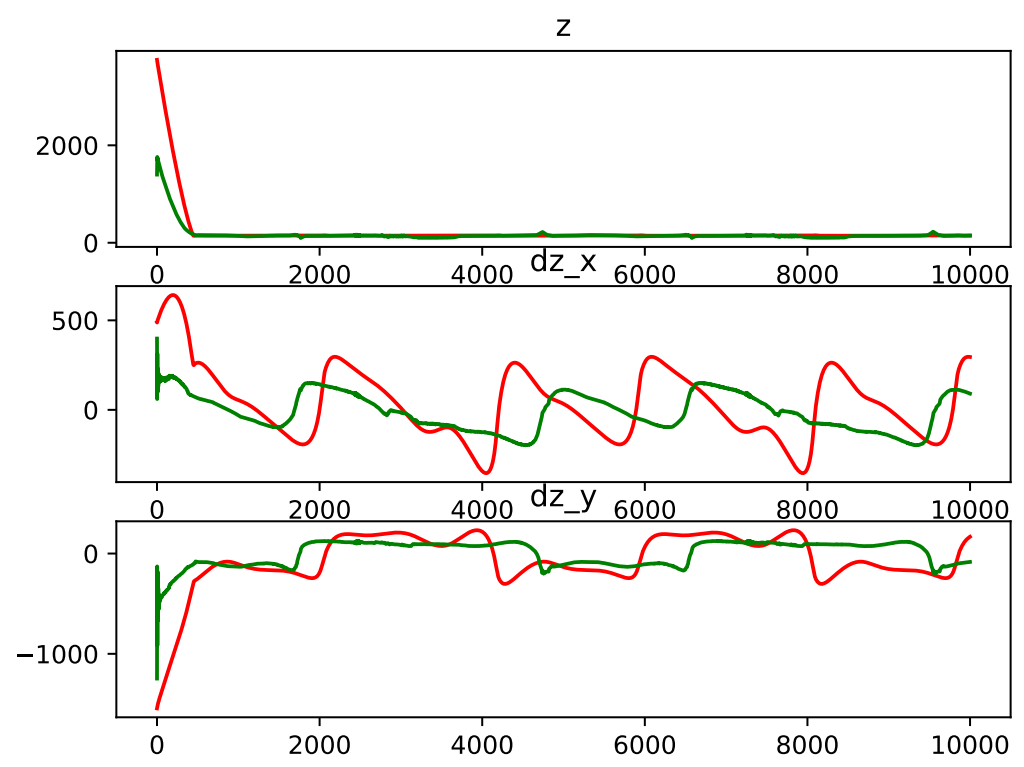

Fig. 16: Field and field gradients for actual and predicted trajectory. $z, d z_{x}$ and $d z_{y}$ represents the field value and field gradients respectively. The $\mathrm{x}$-axis represents iteration number (time-steps) as the formation tracks the level-curve. Red line shows the values followed by traditional cooperative Kalman Filter. Green line shows the values generated using LSTM-KF model. The vertical separation at each time-step shows the error which we try to reduce during LSTM training.

We plot field value $z_{c}$ over the number of iterations in Fig. 17 for various shapes covered in Fig. 15. The plots show how long it takes for the formation to find the level-curve with a desired field value, and how good it tracks the level-curve once the formation is on the level-curve. 

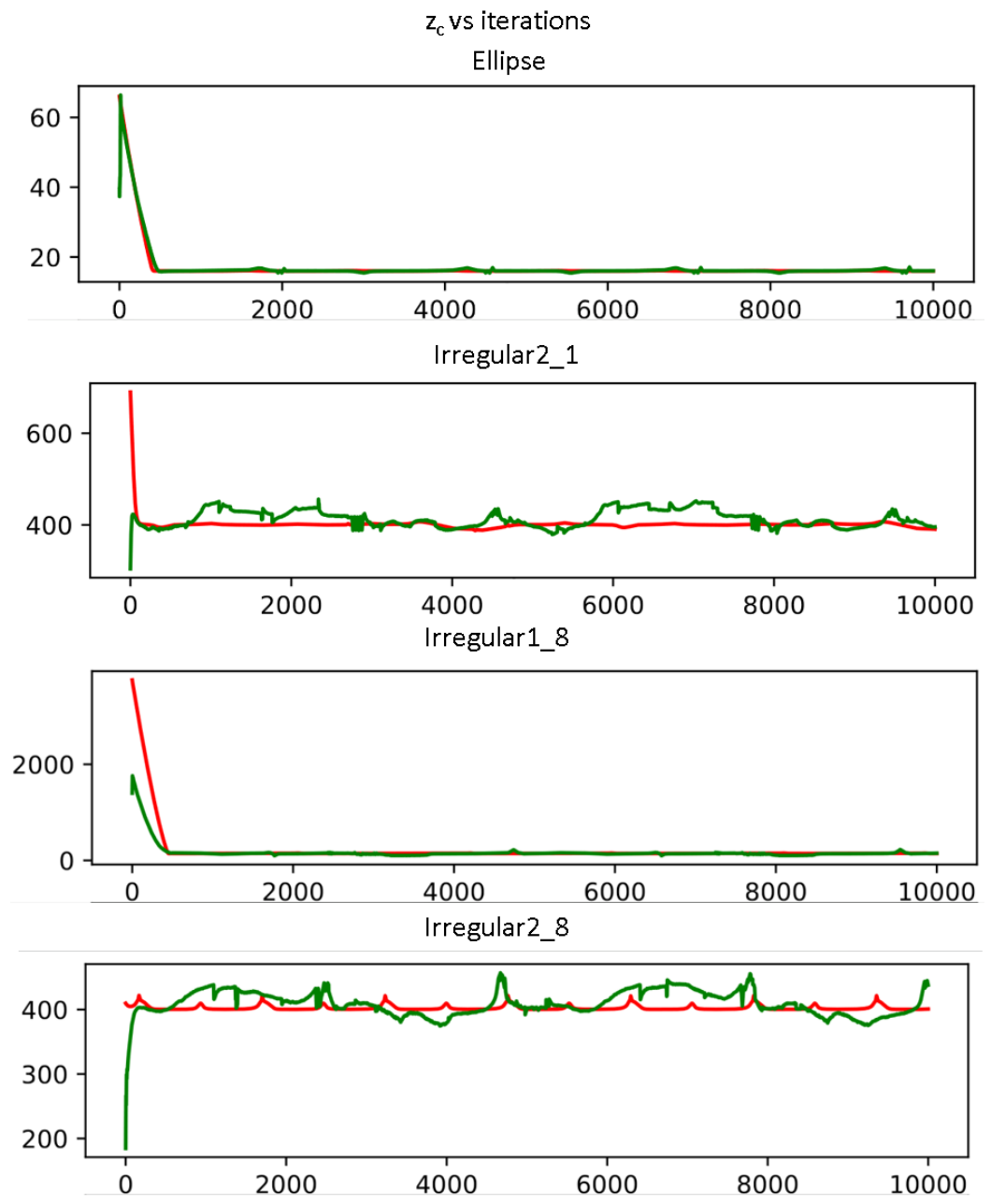

Fig. 17: $z_{c}$ vs number of iterations for actual and predicted trajectory. $z_{c}$ represents the field value at the formation center $r_{c}$. The $\mathrm{x}$-axis represents iteration number (time-steps) as the formation tracks the level-curve. Red line shows the values followed by traditional cooperative Kalman Filter. Green line shows the values generated using LSTM-KF model. The figures show that the formation first approaches the level-curve and then tracks it. For some of the irregular shapes we observe noise because of which the formation deviates from the desired field value. These disturbances correspond to the errors observed in Fig. 15. The trajectory repeats the closed level-curve multiple times over 10k iterations, so we observe periodic behavior. The period for each curve is different.

Next, we show a density plot in Fig. 18 of the field and the field gradients obtained while tracing the ellipse level-curve. This is the distribution on which LSTM is trained. Instead of a machine learning LSTM model, if we want to use traditional time series 
statistical methods like auto regression moving average (ARMA) these densities should have a Gaussian distribution. That is, the series should be stationary (containing no trend/seasonality), which is not the case here. Besides, here we are dealing with multiple time series, so simple ARMA would not suffice, we would have to use vector auto regression (VAR). We considered applying some of these statistical methods, but LSTM offers the greatest flexibility while making minimal assumptions on the input data.

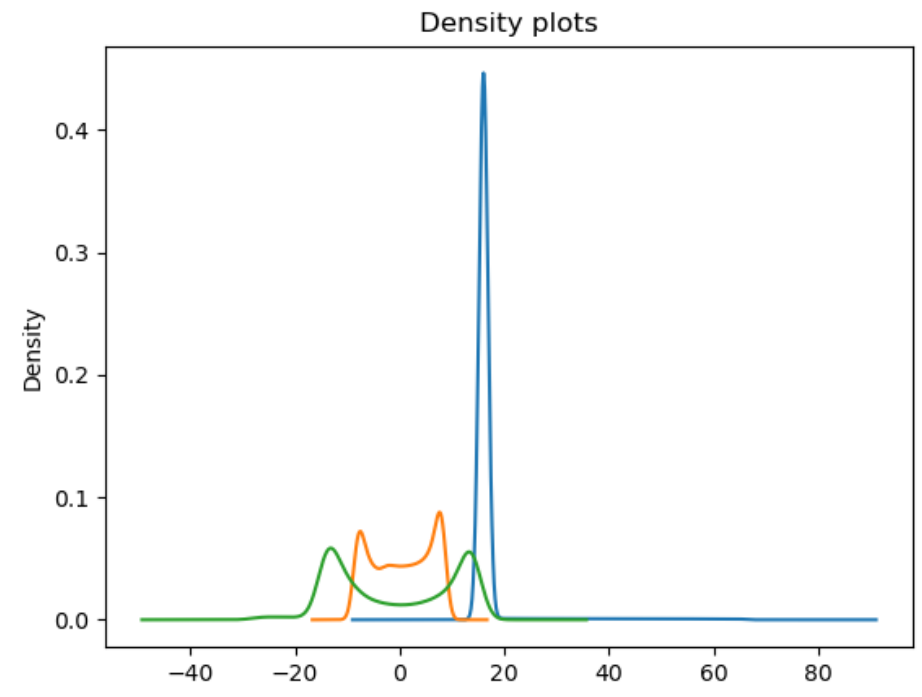

Fig. 18: Density plot for ellipse. The density plot shows the distribution of field $z_{c}$ (blue) and gradient values (green for $\nabla_{x} z_{c}$, orange for $\nabla_{y} z_{c}$ ). The distribution is not Gaussian.

We look at how to decide lag-window hyper parameter. We plot the correlation across lagged values to judge for which values to train the LSTM on, i.e. how far into the history we need to go. For Fig. 19 the more diagonal the plot the higher the correlation. We see that till t-40 there's very high correlation, as we go back in history at t-160 the plot is pretty diffused, i.e. low correlation. 

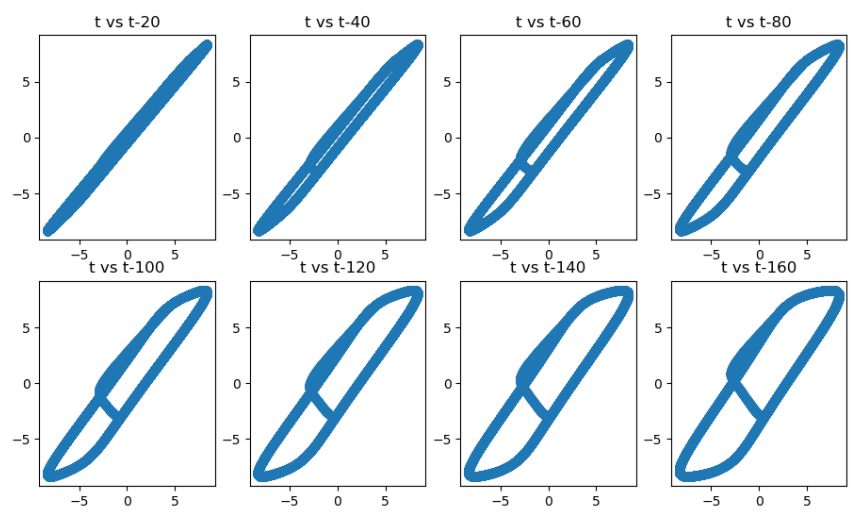

Fig. 19: Correlation with different lags. Each of the 8 plots shows the correlation between the field gradient $\nabla_{x} z_{c}$ at the current time step vs $\nabla_{x} z_{c}$ at $t-t^{\prime}$ time-step. A diagonal plot represents high correlation, while a diffused plot shows no correlation. As we increase the time-lag $t^{\prime}$ we observe vanishing correlation.

Similar information can be obtained from the auto-correlation plot for different lag values (Fig. 20). If the auto-correlation falls into the blue shaded cone it means the correlation can be a statistical fluke. If auto-correlation is outside the cone, there's a high chance that the correlation is not a statistical fluke. We see that until about 400 lags there is detectable auto-correlation for field gradients. The field value doesn't matter much because we are tracing a constant field value, but we see that up to about 200 lags field value has statistical auto-correlation. Note that in our data-set we also have the portion of the trajectory for which the robots have not reached the desired field value, so not all the field values are constant. We can see that in Fig. 16, we start with a high field value and approach the desired field in a limited number of steps.

Using the data from lag-correlation (Fig. 19) and auto-correlation (Fig. 20) plots, we decided to try 50 lag-steps as a good starting point to train LSTM on. That is we provide 50 historical state values to predict the single next state. 


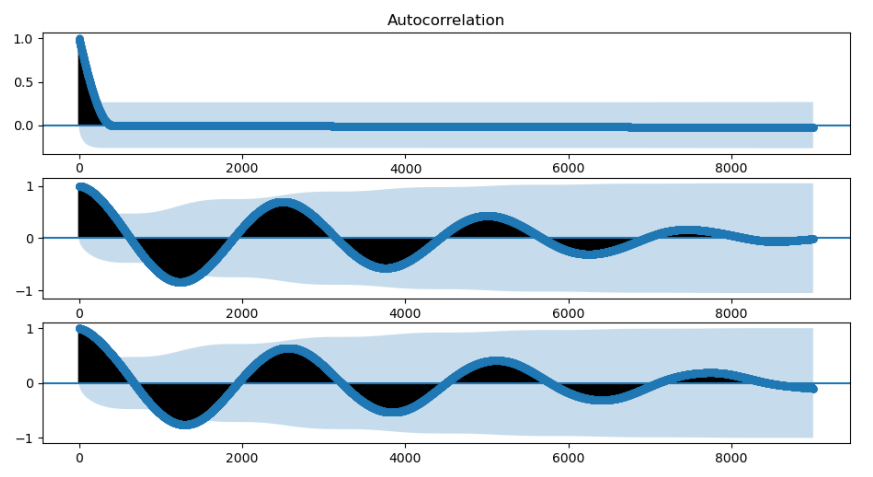

Fig. 20: Auto correlation plot. From top to bottom, the plot shows auto-correlation for $z_{c}\left(1^{s t}\right.$ plot $), \nabla_{x} z_{c}\left(2^{\text {nd }}\right.$ plot $), \nabla_{y} z_{c}$ ( $3^{r d}$ plot $)$ along the $\mathrm{y}$-axis. The $\mathrm{x}$-axis shows lag for auto-correlated sequence. The light blue cone represents the area within which the correlation is a statistical fluke. We observe that for lags $k<200$ the auto-correlation is outside the light blue cone and hence can be modelled.

Finally, we show the results for two robot formation. This state and measurement LSTMs were trained using 4 robot formation. Initially while testing this model for 2 robot formation, the formation would diverge away from the circle. However, after tuning some of the formation-center and formation-shape controller parameters the LSTM model was able to track the circle-shaped level-curve. Through experimentation for 2 robot formation, we find that a lag-window of 4 and 100 neurons in each LSTM layer works the best.

Fig. 21 shows the trajectory taken by 2 robot formation around the circle. As we can see there's some loss in accuracy especially towards the end of the circle, i.e. as we come closer to the starting point. We believe the reason is that LSTM is not able to learn long term relationship very well. We didn't see this in 4 robot formations but we see it in 2 robot formation. We think this is because of the Kalman Filter step in which we update/correct the LSTM estimate. With only 2 robots, it doesn't have enough effect with only 2 measurements in comparison to 4 measurements in the 4 robot case. 


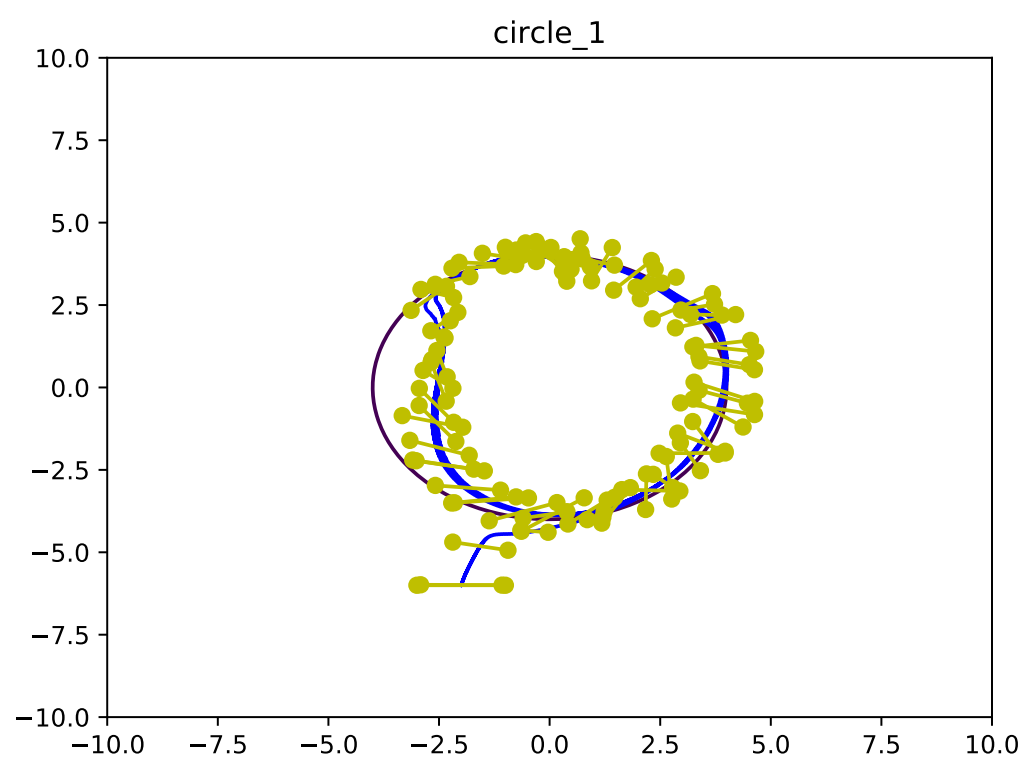

Fig. 21: Trajectory traced by two robot formation around circular level-curve. Yellow dots represent the 2 robot sensors. An imaginary line connects them to the formation center $r_{c}$. Violet curve denotes the real level-curve. Blue shows the trajectory followed by the formation. Here the LSTM-KF model was trained using four robots and tested using two robots.

Fig. 22 shows the field and the field gradient errors for the same trajectory. We see large error for $\mathrm{Y}$ direction gradient $d z_{y}$ when the formation is towards the end of the circle. We also see noisy behavior during this phase. This is because the LSTM output is oscillating in an unstable manner. 


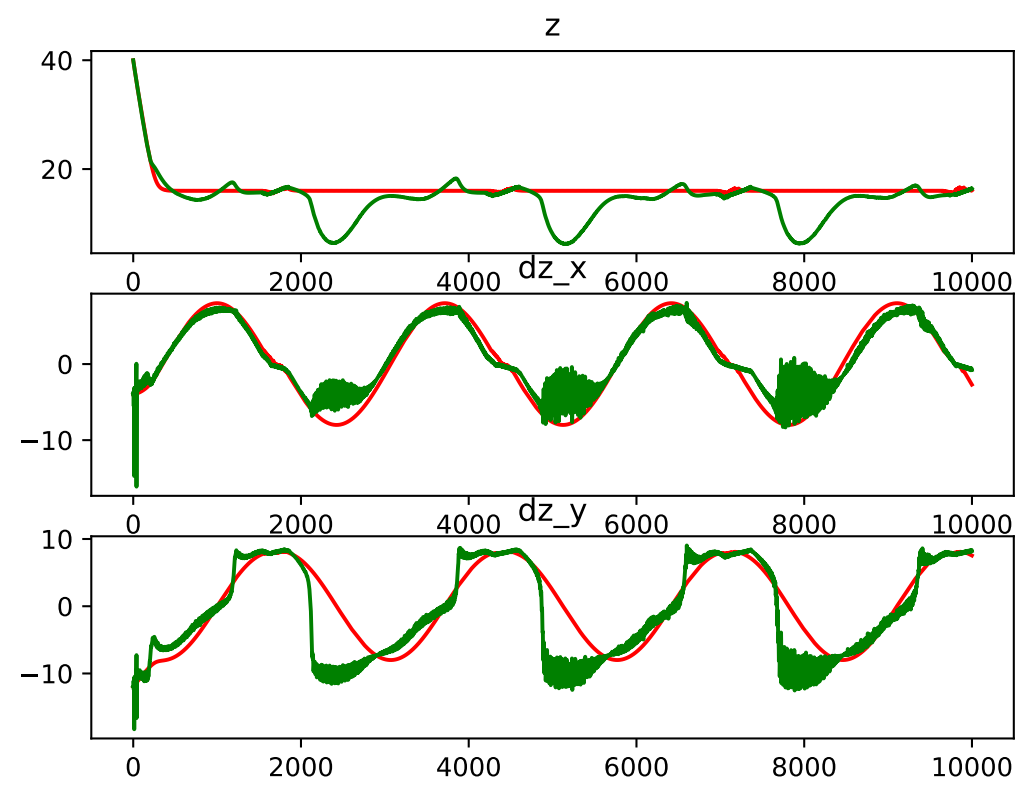

Fig. 22: Field and field gradients for actual and predicted 2 robot formation trajectory. Red line shows the real field and gradient values at $r_{c}$ over iteration count. Green line shows the values predicted by LSTM-KF trained using 4 robots. The disturbance in green lines correspond to the locations at which the formation veers off the level-curve in Fig. 21. 


\section{CONCLUSIONS AND FUTURE WORK}

In this work, we develop a LSTM-enabled cooperative level-curve tracking strategy, which allows a mobile sensor network to detect and track a level-curve in a scalar field with a desired level value. We have shown that using cooperative LSTM-enhanced Kalman Filter allows us to use a sequence of states from history along with sensor measurements to estimate the current state. We have used two LSTMs one for predicting the state and another for predicting the estimation error covariance. We use the sensor measurements in the KF to update the LSTM predictions which are solely based on the sequence of states and errors. By combining these LSTM-KF estimates with the formation center and shape controllers we show that a group of robots using this combined motion control flow tracks the desired level-curve. We show that the LSTM can be trained on a set of known fields and deployed in unknown fields, i.e. the LSTM generalizes well outside of training data. Using a sequence of states with LSTM-KF opens up the possibility to deploy a trained LSTM model into an unknown field using a lower number of robots, while we can still use a larger number of robots to increase the model training accuracy. We show that the LSTM can be trained using four robots and deployed for testing using two robots, the resulting two robot formation tracks the level-curve although with some loss in accuracy. We also show that we can use an LSTM to estimate the hessian of the field which is used by the cooperative LSTM-KF and formation center controller.

In the future, we can improve the accuracy of the LSTM so that the two robot formation tracks the level-curve more closely. For the two robot formation tuned the controller parameters to achieve level-curve tracking, in the future we can consider training these parameters using a machine learning model. We have considered static fields for this work, we can expand it to dynamic or time-varying fields. 


\section{Literature Cited}

[1] D. Culler, D. Estrin, and M. Srivastava, "Overview of sensor networks. comput," in IEEE, August, pp. 41-49, 2004.

[2] S. Li, R. Kong, and Y. Guo, "Cooperative distributed source seeking by multiple robots: Algorithms and experiments," IEEE/ASME Transactions on mechatronics, vol. 19, no. 6, pp. 1810-1820, 2014.

[3] P. Ogren, E. Fiorelli, and N. E. Leonard, "Cooperative control of mobile sensor networks: Adaptive gradient climbing in a distributed environment," IEEE Transactions on Automatic control, vol. 49, no. 8, pp. 1292-1302, 2004.

[4] D. Marthaler and A. L. Bertozzi, "Tracking environmental level sets with autonomous vehicles," in Recent developments in cooperative control and optimization, pp. 317-332, Springer, 2004.

[5] E. E. Rosero García, Cooperative source seeking and level curve tracking for multi-agent systems. PhD thesis, Technische Universität Hamburg-Harburg, 2017.

[6] F. Zhang and N. E. Leonard, "Cooperative filters and control for cooperative exploration," IEEE Transactions on Automatic Control, vol. 55, no. 3, pp. 650-663, 2010.

[7] R. K. Williams and G. S. Sukhatme, "Probabilistic spatial mapping and curve tracking in distributed multi-agent systems," in 2012 IEEE International Conference on Robotics and Automation, pp. 1125-1130, IEEE, 2012.

[8] N. E. Leonard, D. A. Paley, F. Lekien, R. Sepulchre, D. M. Fratantoni, and R. E. Davis, "Collective motion, sensor networks, and ocean sampling," Proceedings of the IEEE, vol. 95, no. 1, pp. 48-74, 2007.

[9] J.-W. Wang, Y. Guo, M. Fahad, and B. Bingham, "Dynamic plume tracking by cooperative robots," IEEE/ASME Transactions on Mechatronics, vol. 24, no. 2, pp. 609-620, 2019.

[10] S. Liu, D. Sun, and C. Zhu, "Coordinated motion planning for multiple mobile robots along designed paths with formation requirement," IEEE/ASME transactions on mechatronics, vol. 16, no. 6, pp. 1021-1031, 2010. 
[11] S. Srinivasan, S. Dattagupta, P. Kulkarni, and K. Ramamritham, "A survey of sensory data boundary estimation, covering and tracking techniques using collaborating sensors," Pervasive and Mobile Computing, vol. 8, no. 3, pp. 358-375, 2012.

[12] M. Choi, R. Sakthivel, and W. K. Chung, "Neural network-aided extended kalman filter for slam problem," in Proceedings 2007 IEEE International Conference on Robotics and Automation, pp. 1686-1690, IEEE, 2007.

[13] Z. Zhang, S. Al-Abri, W. Wu, and F. Zhang, "Level curve tracking without localization enabled by recurrent neural networks," in 2020 th International Conference on Automation, Control and Robotics Engineering (CACRE), pp. 759-763, IEEE, 2020.

[14] A. Jadbabaie, J. Lin, and A. S. Morse, "Coordination of groups of mobile autonomous agents using nearest neighbor rules," Departmental Papers (ESE), p. 29, 2003.

[15] J. P. Desai, J. P. Ostrowski, and V. Kumar, "Modeling and control of formations of nonholonomic mobile robots," IEEE transactions on Robotics and Automation, vol. 17, no. 6, pp. 905-908, 2001.

[16] P. Ogren, M. Egerstedt, and X. Hu, "A control lyapunov function approach to multi-agent coordination," in Proceedings of the 40th IEEE Conference on Decision and Control (Cat. No. 01CH37228), vol. 2, pp. 1150-1155, IEEE, 2001.

[17] F. Zhang, M. Goldgeier, and P. S. Krishnaprasad, "Control of small formations using shape coordinates," in 2003 IEEE International Conference on Robotics and Automation (Cat. No. 03CH37422), vol. 2, pp. 2510-2515, IEEE, 2003.

[18] R. Olfati-Saber and J. S. Shamma, "Consensus filters for sensor networks and distributed sensor fusion," in Proceedings of the 44th IEEE Conference on Decision and Control, pp. 6698-6703, IEEE, 2005.

[19] J. Ma, M. Ye, Y. Zheng, and Y. Zhu, "Consensus analysis of hybrid multiagent systems: A game-theoretic approach," International Journal of Robust and Nonlinear Control, vol. 29, no. 6, pp. 1840-1853, 2019.

[20] W. Wu and F. Zhang, "Cooperative exploration of level surfaces of three dimensional scalar fields," Automatica, vol. 47, no. 9, pp. 2044-2051, 2011. 
[21] J. Brownlee, Introduction to time series forecasting with python: how to prepare data and develop models to predict the future. Machine Learning Mastery, 2017.

[22] G. E. Box and D. R. Cox, "An analysis of transformations," Journal of the Royal Statistical Society: Series B (Methodological), vol. 26, no. 2, pp. 211-243, 1964.

[23] G. E. Box, G. M. Jenkins, and G. Reinsel, "Time series analysis: forecasting and control holden-day san francisco," BoxTime Series Analysis: Forecasting and Control Holden Day1970, 1970.

[24] J. Brownlee, Deep learning for time series forecasting: Predict the future with MLPs, CNNs and LSTMs in Python. Machine Learning Mastery, 2018.

[25] N. Kalchbrenner, I. Danihelka, and A. Graves, "Grid long short-term memory," arXiv preprint arXiv:1507.01526, 2015.

[26] A. Graves, A.-r. Mohamed, and G. Hinton, "Speech recognition with deep recurrent neural networks," in 2013 IEEE international conference on acoustics, speech and signal processing, pp. 6645-6649, IEEE, 2013.

[27] J. Donahue, L. Anne Hendricks, S. Guadarrama, M. Rohrbach, S. Venugopalan, K. Saenko, and T. Darrell, "Long-term recurrent convolutional networks for visual recognition and description," in Proceedings of the IEEE conference on computer vision and pattern recognition, pp. 2625-2634, 2015.

[28] Z. Zhao, W. Chen, X. Wu, P. C. Chen, and J. Liu, "Lstm network: a deep learning approach for short-term traffic forecast," IET Intelligent Transport Systems, vol. 11, no. 2, pp. 68-75, 2017.

[29] D. M. Nelson, A. C. Pereira, and R. A. de Oliveira, "Stock market's price movement prediction with 1stm neural networks," in 2017 International joint conference on neural networks (IJCNN), pp. 1419-1426, IEEE, 2017.

[30] S. X. Yang and C. Luo, "A neural network approach to complete coverage path planning," IEEE Transactions on Systems, Man, and Cybernetics, Part B (Cybernetics), vol. 34, no. 1, pp. 718-724, 2004.

[31] R. G. Krishnan, U. Shalit, and D. Sontag, "Deep kalman filters," arXiv preprint arXiv:1511.05121, 2015. 
[32] C. Doshi, A deep learning approach to state estimation from videos. $\mathrm{PhD}$ thesis, Massachusetts Institute of Technology, 2018.

[33] D. Masti and A. Bemporad, "Learning nonlinear state-space models using deep autoencoders," in 2018 IEEE Conference on Decision and Control (CDC), pp. 3862-3867, IEEE, 2018.

[34] L. Zhang, G. Wang, and G. B. Giannakis, "Real-time power system state estimation and forecasting via deep unrolled neural networks," IEEE Transactions on Signal Processing, vol. 67, no. 15, pp. 4069-4077, 2019.

[35] H. Coskun, F. Achilles, R. DiPietro, N. Navab, and F. Tombari, "Long short-term memory kalman filters: Recurrent neural estimators for pose regularization," in Proceedings of the IEEE International Conference on Computer Vision, pp. 5524-5532, 2017.

[36] Z. Zhang, M. Hou, F. Zhang, and C. R. Edwards, "An lstm based kalman filter for spatio-temporal ocean currents assimilation," in Proceedings of the International Conference on Underwater Networks \& Systems, pp. 1-7, 2019.

[37] fdeloche, "Recurrent neural network unfold," Jun 2017. Accessed on 10.31.2020.

[38] S. Hochreiter and J. Schmidhuber, "Long short-term memory," Neural computation, vol. 9, no. 8, pp. 1735-1780, 1997.

[39] G. Chevalier, “The 1stm cell,” May 2018. Accessed on 10.31.2020.

[40] C. Olah, "Understanding lstm networks." Accessed on 12.07.2019.

[41] K. Cho, B. Van Merriënboer, C. Gulcehre, D. Bahdanau, F. Bougares, H. Schwenk, and Y. Bengio, "Learning phrase representations using rnn encoder-decoder for statistical machine translation," arXiv preprint arXiv:1406.1078, 2014.

[42] A. Graves and N. Jaitly, "Towards end-to-end speech recognition with recurrent neural networks," in International conference on machine learning, pp. 1764-1772, 2014.

[43] I. Sutskever, J. Martens, and G. E. Hinton, "Generating text with recurrent neural networks," in Proceedings of the 28th International Conference on Machine Learning (ICML-11), pp. 1017-1024, 2011. 
[44] A. Graves, "Generating sequences with recurrent neural networks," arXiv preprint arXiv:1308.0850, 2013.

[45] M.-T. Luong, I. Sutskever, Q. V. Le, O. Vinyals, and W. Zaremba, "Addressing the rare word problem in neural machine translation," arXiv preprint arXiv:1410.8206, 2014. 